\title{
Mitteilungen der DGN
}

Nervenarzt 2020 $91: 760-774$

https://doi.org/10.1007/s00115-020-00977-y

○) Springer Medizin Verlag GmbH, ein Teil von Springer Nature 2020

\section{Redaktion}

Prof. Dr. Gereon Nelles, Schriftführer, nelles@dgn.org (v.i.S.d.P.)

Neuromed-Campus Hohenlind, Werthmannstr. 1c, 50935 Köln

Prof. Dr. med. Peter Berlit, Generalsekretär, berlit@dgn.org

Dr. phil. Bettina Albers, Dr. med. Martina Berthold

Prof. Dr. Christine Klein, Präsidentin, klein@dgn.org

Dr. rer. nat. Thomas Thiekötter, Geschäftsführer, thiekoetter@dgn.org

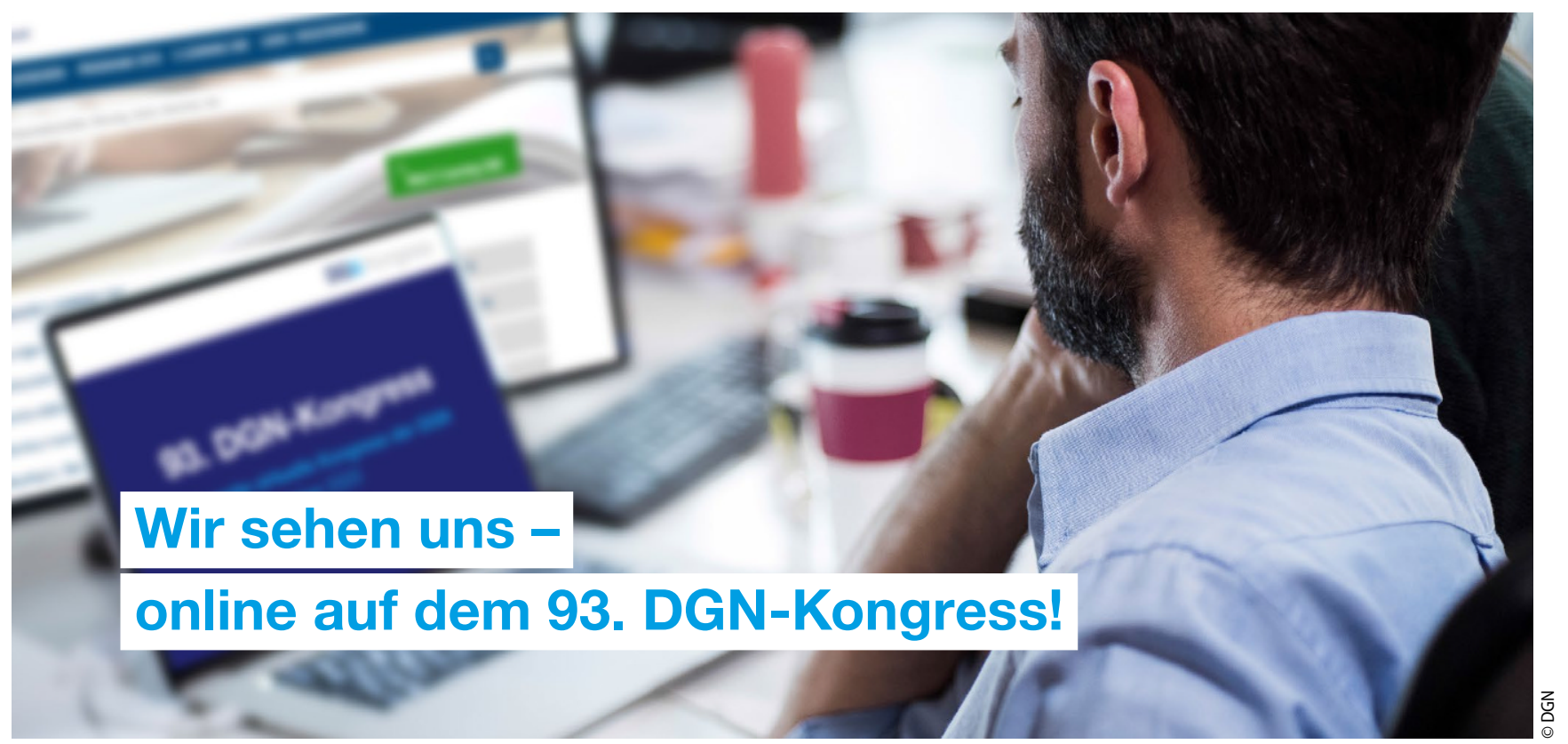

\section{DGN-Kongress im digitalen Exil}

Der DGN-Kongress im digitalen Exil wird neue Maßstäbe setzen. Denn wir verstehen unter einem virtuellen Kongress mehr als nur das Ablegen von Präsentationen auf einer Online-Plattform. Wir werden die digitalen Möglichkeiten voll ausschöpfen, um das, was unseren Kongress ausmacht - die Interaktivität, die Diskussionen, das, ,Wir-sind Neurologie-Gefühl" und "Live"-Erlebnis -, auch im „Corona-Exil“ umzusetzen. Halten Sie sich den 4. November von 14.00-21.30 $\mathrm{Uhr}$, den 5. \&6. November von jeweils 16.00 21.30 Uhr und den 7. November von 09.15-14.00 Uhr frei, um aus der Ferne ganz nah dabei zu sein.

\section{Miterleben! Das Live- Programm}

Das Live-Programm, welches aus Studios in Berlin gesendet wird, besteht aus den drei Hauptkanälen Wissenschaft, Fortbildung und Industrie. Darüber hinaus werden am Kongress-Freitag, den 6. November, die Jungen Neurologen einen vierten LiveKanal bespielen.

Die einzelnen Sitzungen des Live-Programms werden von Top-Experten geleitet, dauern 60 Minuten und beinhalten viele interaktive Elemente wie Votings und Publikumsbewertungen. Für die Kanäle Wissenschaft und Fortbildung sind im Anschluss an jede Session 30 Minuten für Fragen und Antworten (Q\&A) vorgesehen, was eine unmittelbare Interaktion zwischen den Zuschauern und den Experten/ Vorsitzenden ermöglicht. Die Vorsitzenden moderieren den Q\&A-Part, strukturieren die Fragen aus der Online-Community und leiten die Diskussion. Jeder Teilnehmer kann seine Fragen und Anmerkungen virtuell einbringen und sich mit der „Community“ vernetzen. Einzelne Referenten stehen im Anschluss in Einzelchats zur Verfügung.

Das Live-Programm wird technisch hochkarätig produziert, es handelt sich um professionelle Produktionen mit Regisseuren in einem Setting wie bei einer TV-Produktion. Dafür werden von der DGN speziell zwei große Studios in einem Berliner Hotel eingerichtet. Die Vorsitzenden und Referenten sind live vor Ort, was den Vorteil hat, dass eine authentische Gesprächs- und Diskussionsatmosphäre entsteht. Auf diese Weise wird das, was Kongressbesucher ansonsten im Veranstaltungssaal live mitverfolgen, auf den Bildschirm gebracht. Gleichzeitig können die Teilnehmer mitdiskutieren, als wären sie direkt dabei. Das Live-Programm verbindet somit ein nahezu reales Kongresserlebnis mit maximaler Sicherheit: Die Teilnehmer bleiben zu Hause, sind aber dennoch mittendrin im Kongressgeschehen!

Und für die, deren Dienstpläne das „Live-Dabeisein“ nicht zulassen: Natürlich können sie bereits wenige Stunden später alles digital abrufen und sich im Nachgang anschauen.

\section{Wissenschaft Live}

Auf diesem Kanal werden die wissenschaftlichen Sitzungen ausgestrahlt, allen voran die Eröffnungsveranstaltung und das Präsidentensymposium (siehe Folgeseiten). Darüber hinaus gibt es Sitzungen zu den Themen „COVID-19 \& Neurologie“, „Schlaganfall“, „Neuroimmunologie“, „Demenz“, weitere Live-Sessions sind „Highlights 


\section{Highlights der klinischen Neurologie: Neurology in Progress, 5. November 2020, 19.00-20.30 Uhr}

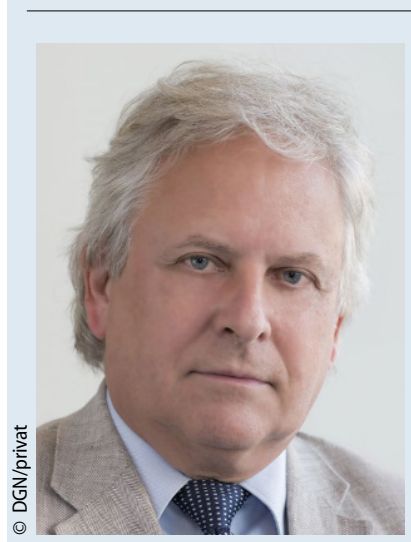

$\Delta$ Prof. Dr. Hans-Christoph Diener

Wissenschaftliche „Druckbetankung", um up to date zu bleiben: DGNPressesprecher Prof. Dr. Hans-Christoph Diener stellt die wichtigsten klinischen Studien in der Neurologie aus dem Zeitraum September 2019 bis Anfang November 2020 vor und erklärt, wie sich die Ergebnisse der Studien in der klinischen Praxis auswirken. Die letzten 30 Minuten sind für Diskussion und Fragen vorgesehen.

\section{Spotlight: Höhepunkte des Kongresses, 7. November 2020, 12.30-14.00 Uhr}

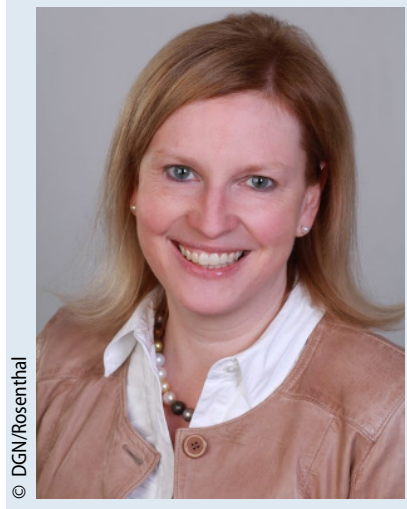

$\Delta$ Prof. Christine Klein, Lübeck, Präsidentin der DGN

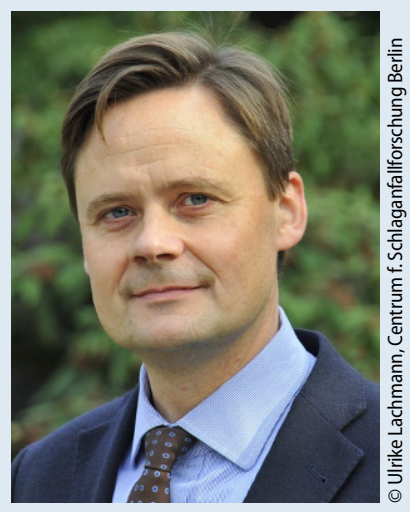

$\Delta$ Prof. Dr. Matthias Endres, Berlin
Wie sagt man so schön - das Beste kommt zum Schluss. Unter dem Vorsitz von DGN-Präsidentin Prof. Dr. Christine Klein, Lübeck, und Kongresspräsident Prof. Dr. Matthias Endres, Berlin, fassen Experten des jeweiligen Fachgebiets die Höhepunkte des wissenschaftlichen Programms zusammen. Folgende Themenschwerpunkte werden von den Experten beleuchtet:

Schlaganfall, Epilepsie, Multiple Sklerose, Bewegungsstörungen, Hirntumore sowie Altern und demenzielle Erkrankungen.

\section{Neurologie und Neurologen im Nationalsozia- lismus, mit anschließender Diskussionsrunde, 6. November 2020, 17.30-19.00 Uhr}

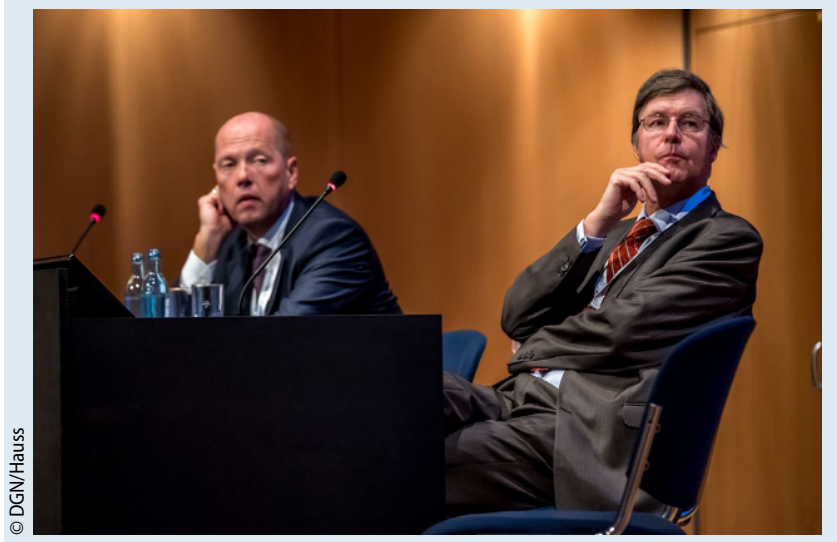

$\Delta$ v. I. n. r.: Prof. Dr. Martin Grond, Prof. Dr. Axel Karenberg

Im Supplement „Neurologen und Neurowissenschaftler in der NS-Zeit ${ }^{\prime}$ (Beilage zu "Der Nervenarzt" 2/2020) wurde die Täterseite beleuchtet und die Vitae von belasteten Persönlichkeiten in der Neurologie aufgerollt. In diesem Symposium soll nun eine Auseinandersetzung mit den Opfern stattfinden. Vortragsthemen sind der „Ausschluss von "nicht arischen" Kolleginnen und Kollegen in der Medizin - diktatorische Maßnahmen und vorauseilender Gehorsam" sowie "Legalisierte Entrechtung - zur juristischen Konstruktion von Entlassung und Vertreibung im Nationalsozialismus". Das Symposium unter dem Vorsitz von Prof. Dr. Martin Grond, Siegen, und Prof. Dr. Axel Karenberg, Köln, lädt zur Diskussion ein.

Im Anschluss sollen auch die Resultate des Moratoriums diskutiert werden, welches Anfang des Jahres beschlossen wurde, um eine sorgfältige historische Aufarbeitung aller Persönlichkeiten, nach denen Wissenschaftspreise und Ehrungen benannt wurden, zu leisten.

der klinischen Neurologie: Neurology in Progress", ein beliebtes Format, in dem Prof. Dr. HansChristoph Diener die wichtigsten klinischen Studien in der Neurologie vorstellt und erklärt, wie sich die Ergebnisse der Studien in der klinischen Praxis auswirken, „Neurologie und Neurologen im Nationalsozialismus" (Informationen dazu siehe oben) und „Spotlight: Höhepunkte des Kongresses“ (siehe links).

Außerdem werden im LiveKongressstrang vier Videoforen präsentiert: Das Veranstaltungsformat des Videoforums ist weit mehr als nur eine mediale, dem Zeitgeist geschuldete Spielerei. Es hat sich durchgesetzt, weil gerade in der Neurologie die phänomenologische Befundung eine große Rolle spielt und Videokasuistiken einen hohen Lehrgehalt haben. Videoforen sind damit innovative Formate der Weiterbildung. 


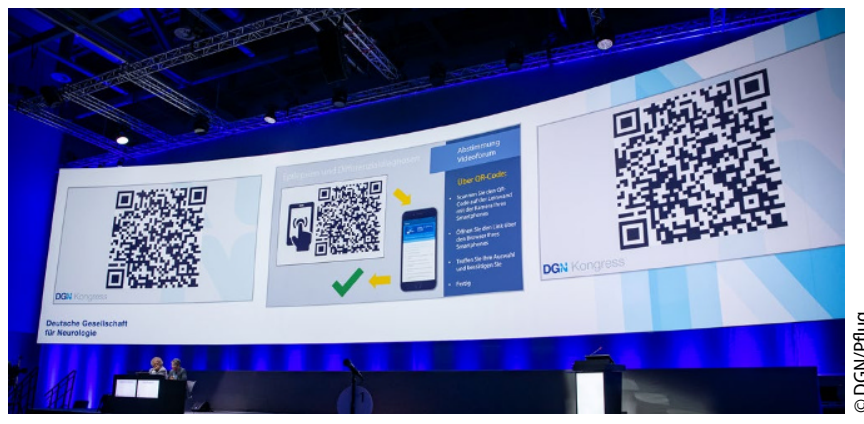

Videoforen

Videoforum Neuromuskuläre

Erkrankungen, 4. November 2020, 17.30-19.00 Uhr

Das Videoforum soll interaktiv eine Auswahl von Fällen genetisch bedingter sowie erworbener, nicht erblicher neuromuskulärer Erkrankungen wie Polyneuropathien, Motoneuronerkrankungen, Myopathien, Muskeldystrophien und lysosomale Speichererkrankungen vor-
Videoforum Schwindel, Okulomotorikstörungen und Nystagmus

- „seeing is believing“,

5. November 2020,

17.30-19.00 Uhr

Bei Augenbewegungsstörungen, Nystagmus und Schwindelsyndromen ist oft buchstäblich eine rasche Blickdiagnose möglich. Deshalb eignet sich dieses Thema auch hervorragend für ein virtuelles Videoforum, um den diagnostischen Blick alias Mustererkennung der Teilnehmer weiter zu schärfen. Die richtige Einordnung dieser Störungen erlaubt oft eine genaue topographische Diagnose: Handelt es sich um eine periphere oder zentrale Läsion im Bereich von Hirnstamm oder Kleinhirn? Interaktiv werden in 90 Minuten deshalb die klinisch relevantesten Befunde dargestellt.

Die Vorsitzenden dieses Videoforums sind Prof. Dr. Michael Strupp, München, Prof. Dr. Dominik Straumann, Zürich/
Schweiz, und PD Dr. Andreas Zwergal, München.

\section{Videoforum Epileptische Anfälle und Differenzialdiagnosen, \\ 6. November 2020, 16.00- \\ 17.30 Uhr}

Es werden Beiträge zu unterschiedlichen Anfallstypen bei Frontallappenepilepsien vorgestellt. Basierend auf der Anfallssemiologie werden der Ort des Anfallsursprungs und ggf. Wege der Anfallspropagation aufgezeigt und diskutiert. Die exemplarischen Anfallsvideos werden im Kontext des jeweiligen Krankheitsbildes präsentiert. Ziel des Videoforums ist das Erkennen von epileptischen Anfällen aus dem Frontallappen und das Verständnis der der Semiologie zugrunde liegenden funktionellen Neuroanatomie.

Die Vorsitzenden dieses Videoforums sind Prof. Dr. Martin Holtkamp, Berlin, und Prof. Dr. Christian G. Bien, Bielefeld.

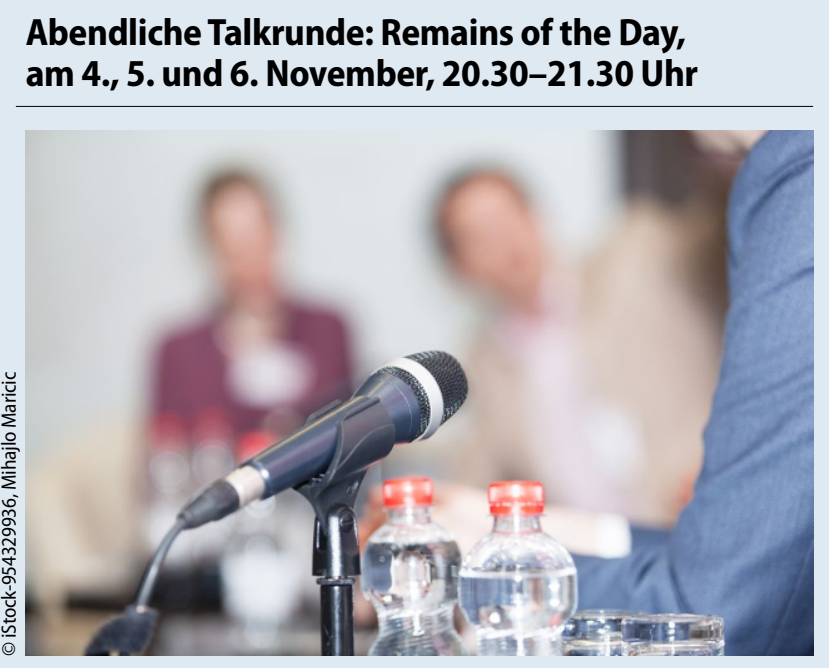

Was vom Tage übrig bleibt: allabendliche Auswertung der in den Live-Sessions vorgestellten Daten und erfolgten Diskussionen fundiert und gleichzeitig höchst unterhaltsam. Genießen Sie dieses Edutainment-Format, bei dem fünf Experten (DGN-Präsidentin Prof. Christine Klein, Stellvertretender DGN-Präsident Prof. Christian Gerloff, Past-Präsident Prof. Gereon Fink, Prof. Martin Grond, Leiter der DGNFortbildungsakademie, und Kongresspräsident Prof. Matthias Endres) zusammen mit einem Gastexperten den Tag auswerten, die Essenz für Klinik und Praxis herausarbeiten und kontrovers diskutieren. Ob Sie die Veranstaltung im „Arbeitsmodus“ im Büro oder als Feierabendevent mit einem Glas Wein auf der Couch verfolgen, bleibt Ihnen überlassen - die Grundbedürfnisse beider Rezeptionsformen, Informationsgewinn und Entertainment, kommen in keinem Fall zu kurz!

\section{Kongress-TV: Neurologie strahlt aus - weit über den Fachkreis!}

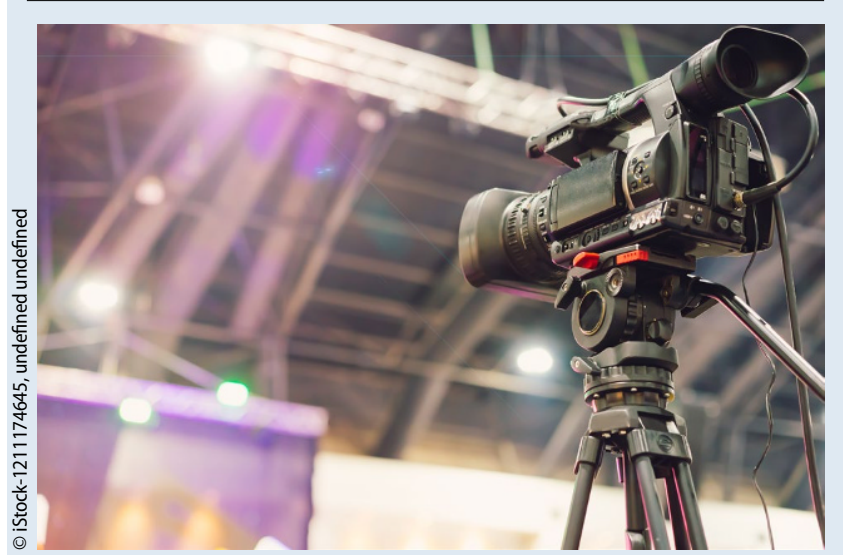

Mit dem Kongress-TV möchten wir neurologische Inhalte über die Fächergrenzen hinaus transportieren: Wir sprechen damit Medizinerinnen und Mediziner anderer Fächer an, Pflegekräfte, Patienten, Angehörige, aber auch Medienmacher und Gesundheitspolitiker. Die Neurologie bietet spannende Themen für die Öffentlichkeit - und wir möchten diese in verschiedenen Formaten aufbereiten: kleine Features, Experteninterviews, Talkrunden - oder der Blick "backstage“ bei einem virtuellen Kongress. Dieser Content ist für alle ohne Log-in zugänglich. 2020, aber auch bei den kommenden Präsenz-Kongressen wird das Angebot um Expertengespräche und kurze Reportagen ergänzt. Federführend wird dieser Kanal durch die Pressestelle der DGN bestückt. 
Videoforum Bewegungsstörungen, 7. November 2020, 11.00-12.30 Uhr

Das Format eines Videoforums bietet sich gerade für das Erlernen/Vertiefen von Diagnosen im Bereich von Bewegungsstörungen an. Eingangs hält Prof. Dr. Andres Ceballos-Baumann ei- nen zehnminütigen Kurzvortrag über das Erkennen von Bewegungsstörungen, dann erfolgen die Videopräsentationen mit interessanten Fallvorstellungen.

Die Vorsitzenden dieses Videoforums sind Prof. Dr. Andres Ceballos-Baumann, München, Prof. Dr. Alexander Münchau,

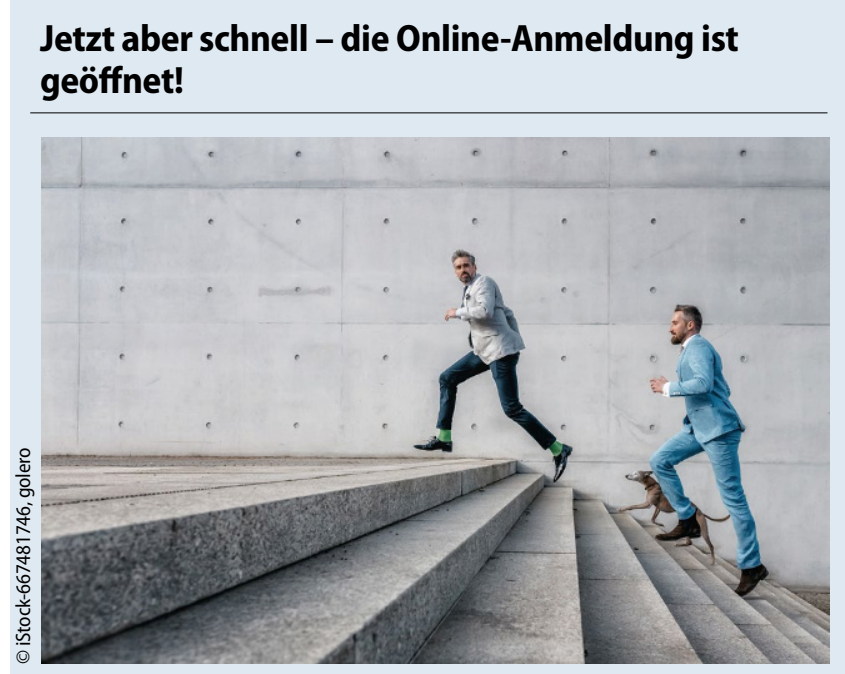

Lust bekommen? Dann halten Sie sich den 4.-7. November 2020 frei und melden Sie sich gleich auf https://www.dgnkongress.org/anmeldung an. Die DGN bietet Ihnen dieses technisch hochwertige, virtuelle Kongresserlebnis zu fairen Teilnahmegebühren. Diese beinhalten:

- alle virtuellen Veranstaltungsteile (Live-Veranstaltungen, Webcasts, E-Poster)

- Zugriff auf Webcasts, E-Poster \& E-Learning für ein Jahr

Willkommen im digitalen Exil - im August ist das Kongressportal online gegangen. Sie können das Programm unter https://www. dgnkongress.org einsehen und sich dort auch gleich zum Kongress anmelden.

DGN-Mitglieder erhalten Anfang September exklusiv ein gedrucktes Programmheft per Post, um sich umfassend über den DGNKongress im digitalen Exil zu informieren.

\begin{tabular}{|c|c|c|c|c|}
\hline \multicolumn{5}{|c|}{ Teilnahmegebühren } \\
\hline & \multicolumn{2}{|c|}{$\begin{array}{l}\text { Frühbucher } \\
\text { (bis 04.10.2020) }\end{array}$} & \multicolumn{2}{|c|}{$\begin{array}{l}\text { Reguläre Preise } \\
\text { (ab 05.10.2020) }\end{array}$} \\
\hline & $\begin{array}{l}\text { DGN- } \\
\text { Mitglied }\end{array}$ & $\begin{array}{l}\text { Nicht- } \\
\text { mitglied }\end{array}$ & $\begin{array}{l}\text { DGN- } \\
\text { Mitglied }\end{array}$ & $\begin{array}{l}\text { Nicht- } \\
\text { mitglied }\end{array}$ \\
\hline Arzt/Ärztin & $75,00 €$ & $130,00 €$ & $90,00 €$ & $150,00 €$ \\
\hline $\begin{array}{l}\text { Assistenz- } \\
\text { arzt/ärztin }\end{array}$ & $50,00 €$ & $80,00 €$ & $75,00 €$ & $120,00 €$ \\
\hline ermäßigt* & $30,00 €$ & $60,00 €$ & $50,00 €$ & $80,00 €$ \\
\hline $\begin{array}{l}\text { Studieren- } \\
\text { de/Pflege }\end{array}$ & - & - & - & - \\
\hline Referenten & - & - & - & - \\
\hline
\end{tabular}

Lübeck, und Prof. Wolfgang $\mathrm{H}$. Oertel, Marburg.

\section{Fortbildung Live}

An allen vier Tagen gibt es analog $\mathrm{zu}$ „Wissenschaft Live“ ein interaktives Fortbildungsprogramm. Über diesen parallelen Live-Kanal werden ausgewählte Kurse der DGN-Fortbildungsakademie angeboten. Die Sessions bestehen aus einem einstündigen Vortragsteil und einer anschließenden, halbstündigen Q\&A-Runde. Die Teilnehmer haben die Möglichkeit, ihre Fragen und Diskussionsbeiträge einzubringen, was eine interaktive Fortbildung ermöglicht. Das Themenspektrum ist breit gefächert und deckt die ganze Bandbreite der Neurologie ab. Das Programm finden Sie auf https://www.dgnkongress.org

\section{Industrie Live}

Auf einem dritten Live-Kanal finden parallel zu den Kanälen Wissenschaft und Fortbildung Live die Industriesymposien statt. Das Programm wird inhaltlich von den Industriepartnern gestaltet. Das Programm finden Sie auf https://www.dgnkongress.org

\section{Junge Neurologen Live}

Am Freitag, den 6. November 2020, bespielen die Jungen Neurologen (JuNos) einen Live-Kanal und bieten Fortbildung von jungen Neurologinnen und Neurologen für junge Neurologinnen und Neurologen bzw. diejenigen, die es werden möchten.

\section{Nachverfolgen! Kongress- inhalte sind bis November 2021 abrufbar}

Neben den Live-Kanälen, die auch im Nachgang angeschaut werden können, gibt es viele zusätzliche Kongressinhalte, die auf der Kongressplattform hinterlegt werden - natürlich ebenfalls hochkarätig produziert und immer auch mit professioneller Videoaufnahme des Sprechers: Denn neuronale Stimulation und Vernetzung ist unser Kerngeschäft!

Diese Webcasts werden ab Kongressbeginn für zwölf Monate „on demand“ zur Verfügung gestellt. Kongressteilnehmer haben also ein Jahr Zugriff auf alle Kongressinhalte inklusive der bereits bewährten E-LearningFormate. Das angebotene Online-Kongressprogramm ist nahezu so umfangreich wie das der vergangenen Präsenzkongresse und kann nach Themen und Referenten durchsucht werden, sodass die Nutzer die gewünschten Inhalte schnell finden.

Das Programm finden Sie auf https://www.dgnkongress.org

\section{Abstracts als E-Poster}

Alle akzeptierten Abstracts werden en bloc ab 4. November 2020 digital veröffentlicht, und zwar in Form eines E-Posters. Die Einreicher haben die Möglichkeit, zusätzlich zu ihren E-Postern eine audiokommentierte PowerPoint-Präsentation einzureichen, die zum Poster online gestellt wird. 


\section{Präsidentensymposium „Neurologie und technologische Innovationen“}

\begin{abstract}
Mit dem Kongressthema "Neurologie und technologische Innovationen" richten Prof. Dr. Matthias Endres, Kongresspräsident, und Prof. Dr. Carsten Finke, Kongresssekretär, die Aufmerksamkeit auf innovative Technologien, die in den vergangenen Jahren bereits wegweisende Erfolge erzielt haben. Die Neurologie und Neurowissenschaften gelten schon lange als Innovationsfach - auf dem Präsidentensymposium am 4. November 2020, 19.00-20.30 Uhr, werden international anerkannte Experten über neueste Entwicklungen in ihrem Fachgebiet berichten, die für Außenstehende noch wie ScienceFiction anmuten und die Faszination für die Neurologie weiter erhöhen werden.
\end{abstract}

Was den Neurowissenschaftler Professor Grégoire Courtine und seine Kollegin, die Neurochirurgin Professor Jocelyne Bloch, antreibt, ist, die Lebensqualität von Patientinnen und Patienten mit schweren Rückenmarkverletzungen zu verbessern. Sie entwickeln am „NeuroRestore“-Zentrum (siehe unten) in Lausanne neurostimulative Ansätze zur Wiederherstellung neurologischer Funktionen. Besondere internationale

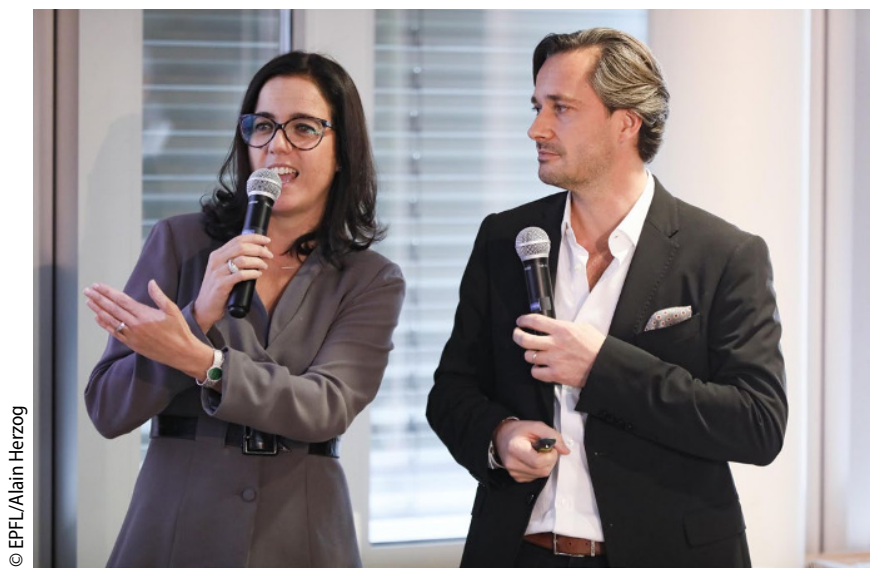

$\Delta$ Ein eingespieltes Team: das Referenten-Duo Professor Jocelyne Bloch (links) und Professor Grégoire Courtine (rechts) aus Lausanne/CH
Beachtung fanden ihre Studien zur Rückenmarkstimulation: Mit einer präzise gesteuerten Stimulation des Rückenmarks wird die Laufbewegung des Patienten unterstützt - querschnittsgelähmte Menschen waren in der Lage, ohne fremde Hilfe einige Schritte zu gehen.

Ähnlich revolutionär ist das Potenzial der tiefen Hirnstimulation, über das Professor Andrea Kühn, Leiterin der Sektion Bewegungsstörungen und Neuromodulation am der Klinik für Neurologie der Charité, berichten wird. Das Verfahren wird bereits erfolgreich bei Morbus Parkinson und Dystonie angewendet, weitere Indikationen im Bereich der Bewegungsstörungen werden erforscht. Nun soll die Stimulation bedarfsgerecht angepasst werden, wobei Hirnsignale als Biomarker dienen, um die sogenannte ClosedLoop-Stimulation zu regulieren. Ende Mai hatte die Deutsche Forschungsgemeinschaft (DFG) die Förderung eines neuen SFB/ Transregio (Berlin, Würzburg) zur Behandlung motorischer Netzwerkstörungen mittels Neuromodulation bekannt gegeben, dessen Sprecherin Prof. Kühn

\section{weniger Behinderungen infolge} von Schlaganfällen.

\section{NeuroRestore - Zukunft "made in Switzerland"}

Die Stiftung Defitech, das Centre hospitalier universitaire vaudois (CHUV), die Biologische und Medizinische Fakultät (FBM) der Université de Lausanne (UNIL) und die École polytechnique fédérale de Lausanne (EPFL) haben beschlossen, ihre Kompetenzen auf dem Gebiet der Neurorehabilitation und der Neurotechnologie, die für neurochirurgische Implantationen erforderlich sind, zu bündeln und im neuen Zentrum Synergien zwischen Ingenieuren, Ärzten und Forschern zu schaffen: Das Zentrum heißt „NeuroRestore". Mit dieser Zusammenarbeit kann die Entwicklung von Elektrostimulationstherapien („Elektrozeutika") zur Verbesserung der motorischen Funktionswiederherstellung bei Paraplegikern, Tetraplegikern, Parkinson- und Schlaganfall-Patienten vorangetrieben werden. Innovative und personalisierte Behandlungen werden im Rahmen von Forschungsprotokollen getestet und dann Krankenhäusern und $\mathrm{Pa}$ tienten zur Verfügung gestellt. Ferner wird es eine der Aufgaben von "NeuroRestore" sein, eine neue Generation von medizinischen Fachkräften und Ingenieuren in der Anwendung dieser innovativen Therapieansätze auszubilden. Für die Leitung von „NeuroRestore“ sind die beiden Professoren Jocelyne Bloch und Grégoire Courtine zuständig, die auf dem Präsidentensymposium einen gemeinsamen Vortrag zum Thema „Restoring neurological functions after spinal cord injury: from mechanisms to patients" halten werden.

Die STIMO-Studie („Stimulation Movement Overground", $\mathrm{Pu}$ blikation vom 1. November 2018 in "Nature"), die vom Neurowissenschaftler Grégoire Courtine von der EPFL und der Neurochirurgin des CHUV, Jocelyne Bloch, durchgeführt wurde, ermöglichte die Entwicklung eines neuen The- 


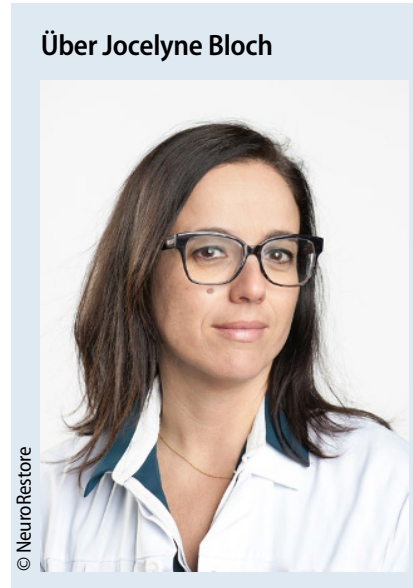

$\Delta$ Professor Jocelyne Bloch

Jocelyne Bloch, Co-Direktorin von "NeuroRestore", ist auch Leiterin des „Programms für stereotaktische funktionelle Neurochirurgie" des CHUV. Nach ihrem Medizinstudium an der Universität Lausanne erhielt sie $2002 \mathrm{ihr}$ Diplom als Neurochirurgin. Sie ist in der translationalen Forschung sehr engagiert und interessiert sich seit Langem für neue therapeutische Indikationen auf dem Gebiet der Neuromodulation und Zelltherapie. Im Jahr 2012 lernte sie Grégoire Courtine kennen. Seither arbeitet sie im Rahmen des STIMO-Projekts daran, Paraplegikern wieder zum Gehen zu verhelfen.

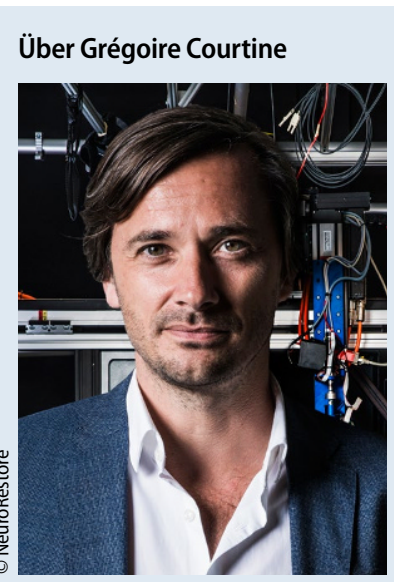

$\Delta$ Professor Grégoire Courtine

Grégoire Courtine, Co-Direktor von "NeuroRestore", leitet auch ein Forschungslabor der EPFL. Er studierte Physik und Neurowissenschaften in Frankreich. Seine Leidenschaft für translationale Neurowissenschaften ist die treibende Kraft hinter seiner Forschungsarbeit zur Entwicklung von Neurotechnologien, die die motorischen Funktionen verbessern sollen. In Los Angeles wurde er als Postdoc von der UCLA ausgezeichnet und 2008 gründete er sein Labor an der Universität Zürich, bevor er zur EPFL wechselte und dort seine Arbeit im Bereich der Paraplegie fortsetzte. Zusammen mit Jocelyne Bloch lancierte er die STIMO-Studie.

rapierahmens für eine bessere neurologische Erholung nach einem Rückenmarktrauma. Die revolutionäre Neurotechnologie kombiniert präzise elektrische, von einem Schrittmacher gesteuerte Stimulationen des Rückenmarks mit einem intelligenten System zur Unterstützung des Körpers. Dank dieser Innovationen waren acht querschnittgelähmte Patienten in der Lage, ohne fremde Hilfe einige Schritte zu machen.

Mittlerweile ist ein Zentrum für die Entwicklung dieser Projekte entstanden. Dank der Unterstützung der Stiftung Defitech und der Zusammenarbeit der EPFL, des CHUV und der UNIL wird das Zentrum „NeuroRestore" die von den beiden Professoren Bloch und Courtine initiierte Forschung vorantreiben, damit den soll. Zwanzig Patienten sollen damit behandelt werden.

\section{Wichtige Information für DGN- Mitglieder zur Ordentlichen Mitgliederversammlung}

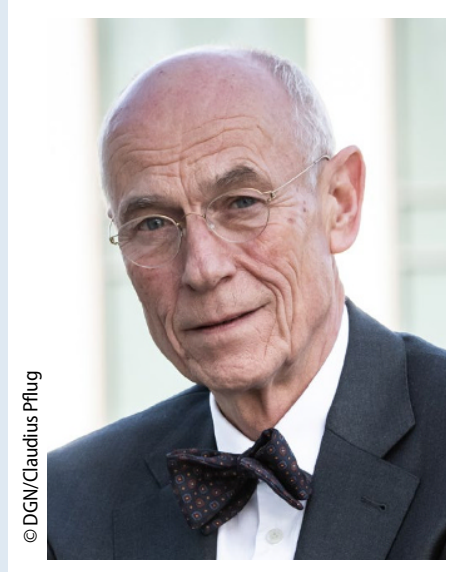

Sehr geehrtes Mitglied, meine Damen und Herren,

wie Ihnen bereits bekannt, findet der diesjährige DGN-Kongress 2020 nicht physisch, sondern virtuell statt. Das DGN-Präsidium hat in seiner Sitzung am 22.06.2020 beschlossen, die ursprünglich für den 04.11.2020 geplante Ordentliche Mitgliederversammlung aufgrund der nicht zu erfüllenden gesetzlichen Vorgaben ersatzlos ausfallen zu lassen. So liegt $z$. B. eine für virtuelle Vereins-Mitgliederversammlungen vorgesehene nachweisbare Anwesenheit von mindestens $50 \%$ aller Mitglieder jenseits realistischer Möglichkeiten. Daraus folgt:

Die satzungsgemäß abzuhandelnden Tagesordnungspunkte der für Anfang November 2020 geplanten ordentlichen Mitgliederversammlung sollen weitgehend durch schriftliche Berichte der jeweiligen Präsidiumsmitglieder ersetzt werden (Bericht der Präsidentin, Bericht des Schatzmeisters und der Wirtschaftsprüfung sowie Bericht der Kassenprüfer). Sämtliche durch die Mitgliederversammlung abzustimmenden Beschlüsse (u.a. die Entlastung der Mitglieder des Präsidiums) werden auf die
Mitgliederversammlung im November 2021 vertagt. Hierunter fällt auch die Wahl eines neuen Stellvertretenden Präsidenten (m/w/d), während der Wechsel von Präsidentin zu Past-Präsidentin und der des amtierenden Stellvertretenden Präsidenten zu Präsident bereits durch die Mitgliederversammlung vom 30.10 .2018 bestätigt wurde und damit zum 01.01.2021 in Kraft treten kann. Schatzmeister und Schriftführer wurden bei dieser Gelegenheit 2018 ebenfalls für weitere zwei Jahre im Amt bestätigt.

Um die für ein Jahr folgerichtig durch die nicht stattfindende Wahl (Stellvertretender Präsident) entstehende Lücke im geschäftsführenden Vorstand (Präsident, Stellvertretender Präsident, Past-Präsidentin) zu kompensieren, hat das DGN-Präsidium mit Wirkung ab dem 01.01.2021 bis zur Wahl eines Nachfolgers des Stellvertretenden Präsidenten bei der nächsten ordentlichen Mitgliederversammlung Herrn Prof. Dr. G. R. Fink zum kommissarischen Stellvertretenden Präsidenten bestimmt. Diese Kooptation stellt keine Wiederwahl im Sinne von $₫ 8$, Ziff. 5 der Satzung dar, sondern ist eine „vorübergehende Selbstergänzung “ des Präsidiums, bei der auch die Amtszeit von vorneherein auf ein Jahr begrenzt ist und welche zur Aufrechterhaltung der vollständigen Handlungsfähigkeit des Vorstands gedacht ist.

Den vollständigen Text dieses Beschlusses finden Interessierte auf www.dgn.org im internen Bereich.

Mit freundlichen Grüßen

\section{Dr. Thomas Thiekötter} Geschäftsführer 


\section{„Neurologen und Neurowissenschaftler in der NS-Zeit": Wie kann die Aufarbeitung eines belasteten Erbes zeitgemäß gelingen?}

\section{Nehmen Sie aktiv am Diskurs teil!}

2015 hat die DGN ein auf sechs Jahre angelegtes Forschungsprojekt angestoßen und renommierte Medizinhistoriker damit beauftragt, die Verstrickung der Fachgesellschaft bzw. einzelner Gesellschaftsmitglieder in das NSRegime aufzuarbeiten: Prof. Axel Karenberg, Köln, Prof. Heiner Fangerau und Dr. Michael Martin, Düsseldorf, von den jeweiligen Instituten für Geschichte und Ethik der Medizin dieser Universitäten haben eine umfassende Publikation

vorgelegt, die im Februar als Supplement in "Der Nervenarzt" ausgeliefert wurde.

Liebe Kolleginnen und Kollegen,

konfrontiert mit den historischen Fakten, stehen wir nun vor der Aufgabe der Aufarbeitung und müssen uns überlegen, wie sie zeitgemäß gelingen kann. Im ersten Schritt haben wir alle Wissenschaftspreise, die mit Eigennamen benannt sind, bis auf Weiteres

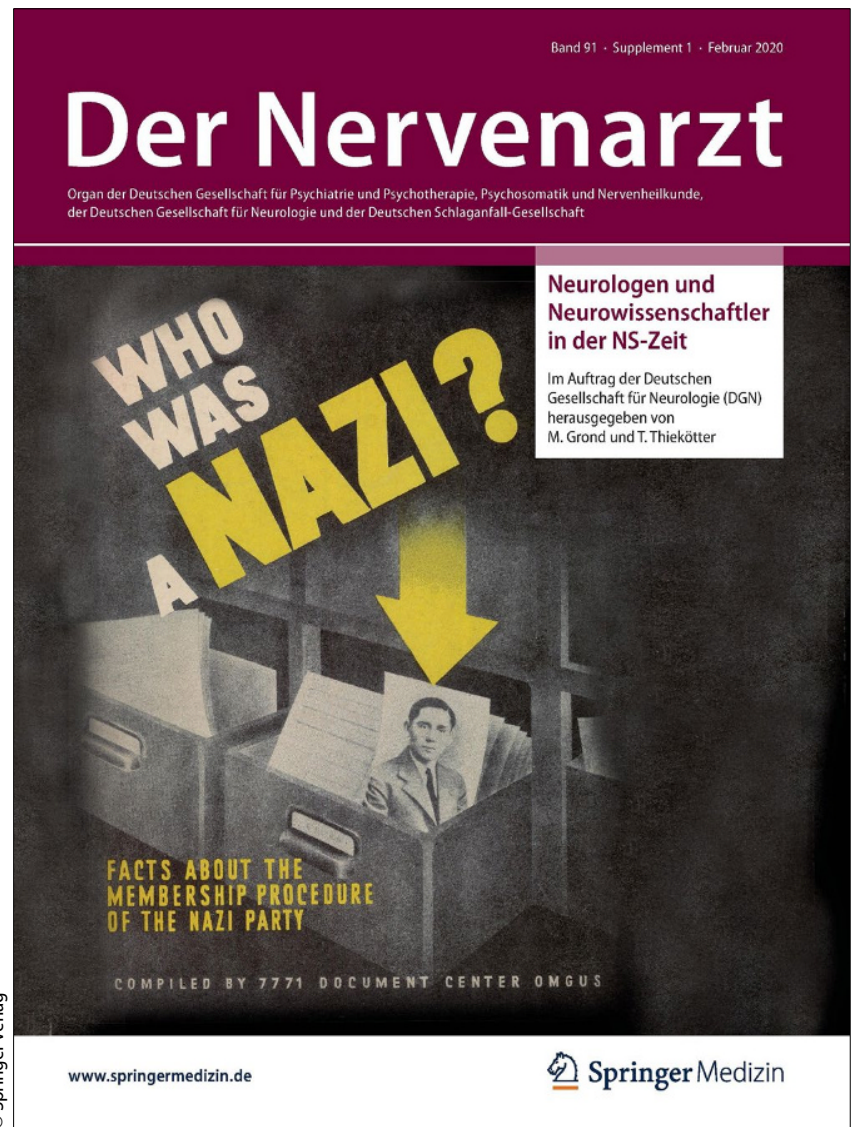

$\Delta$ Cover des Supplements "Neurologen und Neurowissenschaftler in der NS-Zeit" ausgesetzt und nutzen das Moratorium für eine sorgfältige historische Aufarbeitung - das Resultat stellen wir auf unserer virtuellen Jahrestagung zur Diskussion.

Erfreulicherweise hat das Supplement auch zu einem lebendigen Diskurs innerhalb unserer Fachgesellschaft geführt - in der Juni-Ausgabe des „Der Nervenarzt" wurden verschiedene Leserbriefe von Mitgliedern veröffentlicht, die eine ganze Bandbreite an Reaktionen zeigen: von erleichterten Beiträgen - „endlich eine längst überfällige Aufarbeitung “ - über einen Rehabilitationsversuch eines von Historikern als belastet eingestuften Neurologen bis hin zu Stimmen, die Parallelen zur heutigen Situation aufzeigen, in der Ärztinnen und Ärzte auch nicht immer nach bestem Wissen und Gewissen handeln könnten: zwar unterlägen sie keinen politischen, aber gravierenden, die ärztliche Freiheit limitierenden, ökonomischen Zwängen.

Wir möchten diese Diskussion fortsetzen und laden Sie ein,
Ihre Gedanken zu Papier zu bringen. Welche Kommentare haben Sie zu dem Forschungsprojekt und $\mathrm{zu}$ den bereits veröffentlichten Leserbriefen? Leserbriefe, die uns bis Ende August erreichen, werden im Oktober-Heft von „Der Nervenarzt“ publiziert werden, spätere Einsendungen in den Folgeausgaben. Bitte senden Sie Ihre Beiträge an presse@ dgn.org.

Interessante Denkansätze und Diskussionsbeiträge werden wir darüber hinaus bei einem Symposium zu diesem Thema auf dem virtuellen DGN-Kongress aufgreifen und mit Ihnen diskutieren.

Als DGN-Mitglied haben Sie die Möglichkeit, den Diskurs aktiv mitzugestalten. Nehmen Sie die Chance wahr - wir freuen uns auf Ihren Beitrag!

Herzlichen Dank

Ihre

Prof. Christine Klein

Präsidentin der DGN

\section{Neue Sonderforschungs- bereiche der DFG: Die Neurologie ist prominent vertreten!}

\section{Deutsche \\ Forschungsgemeinschaft}

Die Deutsche Forschungsgemeinschaft (DFG) hat Ende Mai die Förderung von zehn neuen Sonderforschungsbereichen (SFB) bekannt gegeben - mit einem Gesamtfördervolumen von 114 Millionen Euro. Unter den zehn neuen SFB sind vier aus dem Bereich der Medizin - und die Neurologie ist darunter mit zwei SFB prominent vertreten.

„Für die DGN ist die DFG-Fördervergabe ein großer Erfolg, denn sie illustriert den hohen Stellenwert der Neurologie innerhalb der Medizin und verankert sie als medizinisches Schlüsselfach", erklärte Professor Dr. Peter Berlit, Essen, Generalsekretär der DGN. Die beiden ausgewählten neurologischen Projektanträge zeichnen sich durch eine hohe Innovationskraft aus und haben zudem Strahlkraft über die Fächergrenze hinaus. „Die innovativen An- 


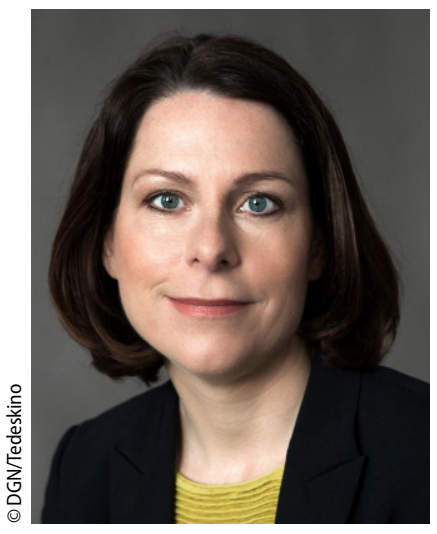

$\Delta$ Prof. Dr. Ulrike Bingel, Essen

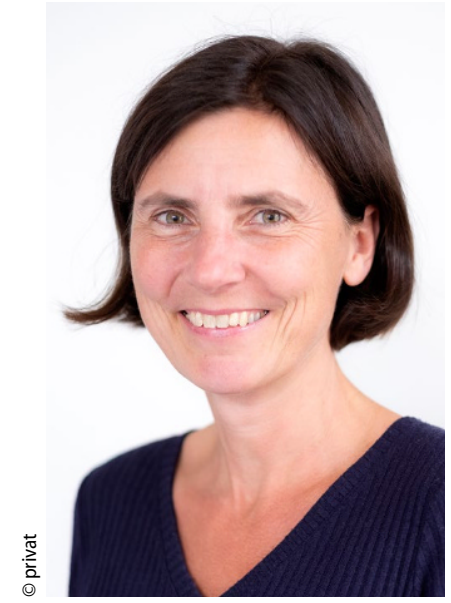

^ Prof. Dr. Andrea Kühn, Berlin

sätze aus der Neurologie werden auch anderen medizinischen Fächern zugutekommen."

Der SFB/Transregio (Essen, Hamburg, Marburg) „Der Einfluss von Erwartung auf die Wirksamkeit medizinischer Behandlungen“, der mit rund zwölf Millionen Euro gefördert wird, erforscht Placebo- und Nocebo-Effekte. „Unser Ziel ist, Erkenntnisse für eine systematische Anwendung von Erwartungseffekten im Rahmen von medizinischen Behandlungen zu generieren“, erklärt die SFBSprecherin Professor Dr. Ulrike Bingel, Essen. An zwei Erkrankungen, Schmerzerkrankungen und affektiven Störungen, soll mit experimentellen Modellen untersucht werden, welche psychologischen und neurobiologischen Mechanismen den Effek- ten von positiven und negativen Behandlungserwartungen $\mathrm{zu}-$ grunde liegen und wie diese Einfluss auf die medikamentöse Therapie nehmen. Ein besonderer Fokus soll dabei auf die Identifikation von Prädiktoren für interindividuelle Unterschiede gelegt werden - „kurz gesagt, uns interessiert, warum eine Therapie bei Patient A wirksam ist, aber trotz gleicher Voraussetzungen bei Patient B versagt", so die Expertin. „Und wir gehen noch einen Schritt weiter: In ersten klinischen Proof-of-ConceptStudien möchten wir untersuchen, wie Erwartungseffekte für die Therapie von Patienten mit chronischen Schmerzen oder Depression nutzbar gemacht werden können. “ Dies wird jetzt in Essen, Hamburg und Marburg in einem interdisziplinären Team aus Medizinern, Psychologen und Grundlagenwissenschaftlern erforscht.

Im Mittelpunkt des zweiten neuen SFB/Transregio (Berlin, Würzburg) aus dem Bereich der Neurologie, der mit über zehn Millionen Euro gefördert wird, steht die Behandlung motorischer Netzwerkstörungen mittels Neuromodulation. „Basierend auf den Erkenntnissen zur tiefen Hirnstimulation bei Patienten mit M. Parkinson, wollen wir die Neuromodulation als ein vielversprechendes Werkzeug zur individualisierten Therapie von Bewegungsstörungen weiterentwickeln. Grundlegende Idee ist, bedarfsgerecht pathologische Netzwerkaktivitäten zu unterdrücken und physiologische zu verstärken, um so Krankheitssymptome zu mildern und Nebenwirkungen zu vermeiden", erklärt SFB-Sprecherin Professor Dr. Andrea Kühn von der Charité Berlin. Ziel ist zunächst die effektive Behandlung motorischer Störungen, wobei das grundlegende Prinzip der Neuromodulation auf andere Hirnerkrankungen wie Schlaganfall, Trauma oder andere neurodegenerative Erkrankungen übertragen werden soll. Um das zu erreichen, müssen von moleku- laren und zellulären Effekten der Neuromodulation bis zur systemphysiologischen Ebene der neuronalen Schaltkreise die Interaktionen erforscht und mittels computergestützter Modellierung Netzwerkeffekte simuliert werden. Diese Forschungsarbeiten sollen in einem interdisziplinären Team aus Medizinern, Neurowissenschaftlern und Grundlagenforschern in Berlin und Würzburg gemeinsam mit weiteren externen Partnern umgesetzt werden.

Ein weiterer SFB/Transregio, „Lokale Kontrolle der Schilddrüsenhormonwirkung (LocoTact)“, untersucht die lokale Kontrolle der Schilddrüsenhormonwirkung und will klären, wie eine fehlerhafte Kontrolle Erkrankungen von Herz, Leber oder Zentralnervensystem begünstigen kann (Universität Duisburg-Essen, Sprecherin: Prof. Dr. Dagmar Führer-Sakel, ebenfalls antragstellend: Charité Berlin - FU Berlin und HU Berlin, Universität Lübeck). Das endokrinologische Projekt hat somit auch eine neurologische Komponente.

„Wir sind sehr stolz auf die Innovationskraft unseres Fachs und ich möchte den beiden Kolleginnen, Prof. Bingel und Prof. Kühn, auch im Namen des DGNPräsidiums herzlich $\mathrm{zu}$ diesem Erfolg gratulieren. Neurologie ist ein Zukunftsfach und in vielerlei Hinsicht am Puls der Zeit. Das zeigt sich nicht zuletzt auch daran, dass die beiden neuen, rein neurologischen SFB von Neurologinnen geleitet werden“, kommentierte Prof. Berlit.

\section{„Neurological Research and Practice" - ein Rückblick auf die ersten 1,5 Jahre}

Ende Februar 2019 ging die erste Ausgabe von „Neurological Research and Practice" online. Ein großer Erfolg für die Deutsche Gesellschaft für Neurologie (DGN), die sich mit diesem englischsprachigen Open-Access-Journal auch international als Motor für Forschung und Innovation präsentiert. Wir sprachen mit dem Herausgeber, Prof. Dr. med. Dr. h.c. Dipl.-Psych. Werner Hacke, darüber, wie sich die junge Fachzeitschrift entwickelt hat, was DGN-Mitglieder machen können, um die positive Entwicklung weiter zu unterstützen, und wo er das Journal in fünf Jahren sieht.

Wie hat sich die "Neurological Research and Practice" in den letzten 1,5 Jahren entwickelt?

Im Prinzip bin ich sehr zufrieden. Die Zeitschrift hat sich gut entwickelt und wir konnten sogar mehr Arbeiten publizieren, als ich ursprünglich geplant hatte. Am Anfang war der Eingang von Manuskripten noch sehr verhalten und hat viel persönliches Engagement abverlangt, ich musste die Kolleginnen/Kollegen persönlich ansprechen, immer wieder einladen und erinnern. Das hat sich aber gelohnt! Wir haben vor Kurzem das 100. Manuskript erhalten - inzwischen liegen wir bei ca. 110 eingereichten Beiträgen. Die meisten Einreichungen kommen aus Deutschland, im Moment noch wenige aus dem Ausland. Im ersten Jahr konnten wir über 45 Beiträge publizieren und 2020 wird sich diese Zahl sicher noch einmal erhöhen. Grundsätzlich unterliegen alle Beiträge einem Peer-Review-Verfahren. Wir haben eine Ablehnungsquote von über $40 \%$, weil wir von Anfang an ein hohes Qualitätsniveau etablieren wollten, und ich bin froh, dass wir mittlerweile genügend Manu- 


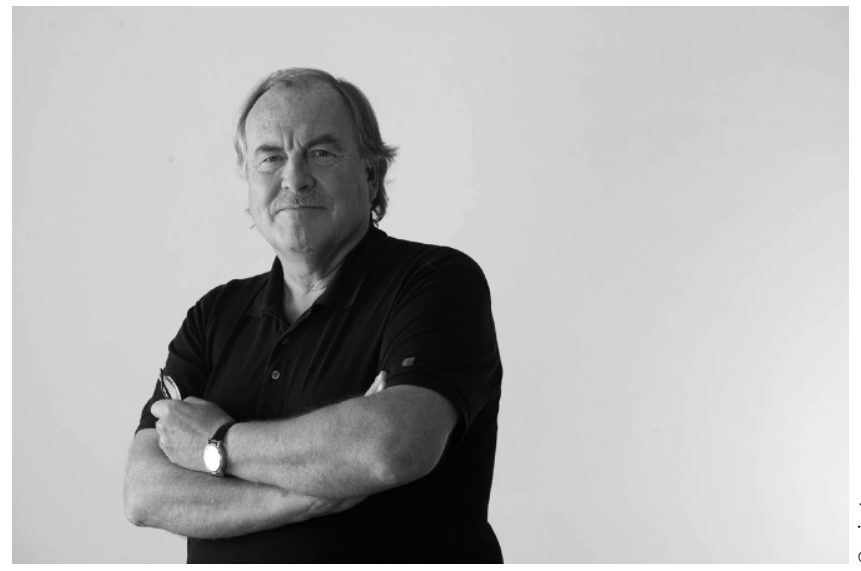

$\Delta$ NRP-Herausgeber Professor Dr. med. Dr. h.c. Dipl.-Psych. Werner Hacke, Heidelberg

skripte erhalten, aus denen wir die besten auswählen können.

Wir können auf eine ganze Reihe an gut zitierten Beiträgen schauen und die thematische Breite dessen, was eingegangen ist, ist ausgesprochen repräsentativ für das Fach. Aber natürlich ist noch eine weitere Steigerung der Einreichungen nötig und möglich!

Wie messen Sie den Erfolg der einzelnen Artikel?

Bewertungstools wie der "Altmetric Attention Score“ greifen bei einer so jungen Zeitschrift natürlich nicht wirklich, sodass wir uns an Aufrufzahlen und Zitierungen orientieren. Viele Artikel, mittlerweile sicher 30 bis 40, haben Zugriffszahlen von über 2000 oder sogar 3000. Ein paar Arbeiten waren so innovativ, dass sie international zitiert wurden und werden. Das trägt natürlich zu einem höheren Bekanntheitsgrad der Zeitschrift bei.

\section{Welche Artikelformate sind vor- herrschend?}

Die Mehrzahl der Artikel sind Originalpublikationen, also Research-Artikel, gefolgt von Reviews und SOPs bzw. Leitlinien. Wir haben auch "Letters" und "Clinical Trial Protocols“, ich denke, die Mischung ist sehr ausgewogen.

Hat die SARS-CoV-2-Pandemie etwas an der Publikationsfreude Ihrer Kolleginnen und Kollegen geändert?
Nein, nicht wirklich. Anfangs, in der Akutphase des Lockdowns war die Befürchtung, dass die Situation in den Kliniken allen so viel abverlangt, dass keine Zeit für die wissenschaftliche Aufarbeitung war. Aber die Situation in den Kliniken hat sich in Deutschland relativ schnell wieder erholt und die neurologischen Auswirkungen des neuartigen Virus bieten nun ein breites Themenspektrum für Publikationen. Wie viele andere Zeitschriften haben auch wir einen „fast track“ für Publikationen zu COVID-19 eingerichtet, aber dennoch unterziehen wir diese Arbeiten einem Peer-Review, worauf im Moment viele Fachzeitschriften verzichten, sogar renommierte. Wir hingegen haben ein beschleunigtes Verfahren eingerichtet, feste Reviewer stehen für diese Themen "stand by “ und bearbeiten die Manuskripte sehr schnell. So hoffen wir, weiterhin eine hohe Qualität halten zu können.

Wo hat die Zeitschrift noch Raum für Entwicklung? Welchen Wunsch haben Sie als Herausgeber der Zeitschrift?

Wo wir gerade beim Thema Review-Prozess sind ... In der Tat habe ich manchmal Probleme, genügend hochqualifizierte Reviewer zu verpflichten, gerade wenn es sich um sehr spezialisierte Themen handelt und die Auswahl der Experten begrenzt ist. Neben der offiziellen Einladung über den „Editorial Manager“ muss ich häufig persönlich nachhaken und die Kolleginnen/Kollegen überzeugen, sich einzubringen, und nicht selten erhalte ich auch Absagen. Ich würde mir in der Tat wünschen, dass die Bereitschaft, aktiv mitzuarbeiten und die Zeitschrift gemeinsam zum Erfolg zu führen, höher wäre.

Wie ist die internationale Resonanz und was können DGN-Mitglieder machen, um die Sichtbarkeit des Titels zu erhöhen?

Die internationale Resonanz entwickelt sich nur langsam, denn natürlich sind wir mit unserer Zeitschrift noch nicht in Pubmed gelistet und haben keinen Impact Factor. Daher wird das Journal international natürlich noch nicht so stark wahrgenommen. Wir arbeiten daran, dass sich das ändert, aber die Aufnahme in Pubmed wird sicher noch ein Jahr dauern und bevor wir einen Impact Factor erhalten, wird sicher noch mehr Zeit ins Land gehen. Umso wichtiger ist es, eigeninitiativ auf Artikel aufmerksam zu machen. Wenn ein Beitrag zu meinen Fachgebieten erscheint, sende ich den Link oder das PDF an einen großen Verteiler internationaler Kolleginnen und Kollegen. Schön wäre, wenn die Autorinnen und Autoren, auch die Reviewer, das ebenso täten. Wichtig sind darüber hinaus heutzutage die sozialen Medien - und natürlich wäre es wünschenswert, wenn viele DGNMitglieder die Artikel teilen, liken oder kommentieren!

Wo sehen Sie "Neurological Research and Practice" in fünf Jahren?

Das kann ich in aller Kürze auf den Punkt bringen: immer noch auf dem Markt, in Pubmed und mit einem Impact Faktor von 2 bis 2,5. Mit der Unterstützung der DGN-Mitglieder halte ich das für durchaus realistisch!

\section{Das Interview führte}

Dr. Bettina Albers

\section{Neue DGN-Leitlinien-Updates}

Die Leitlinienarbeit ist eine wesentliche Aufgabe der DGN. Bislang sind über 90 Leitlinien erschienen und werden regelmäßig aktualisiert. Leitlinien umfassen eine ausführliche Literaturrecherche und Einordnung neuer Arbeiten. Die komplexen Abstimmungsprozesse mit zahlreichen Experten sowie verschiedenen Fachgesellschaften dauern oft viele Monate. Die rund 900 ehrenamtlich tätigen Autoren und die Redaktion unter der Leitung von Prof. Helmuth Steinmetz, Frankfurt am Main, Sprecher der Kommission Leitlinien, und Prof. Dr. Hans-Christoph Diener, Essen, stellvertretener Sprecher, bemühen sich, Aktualisierungen so schnell wie möglich auf den Weg zu bringen. Auf diese Weise kommen neue wissenschaftliche Erkenntnisse schneller den Ärzten und schließlich den Patienten zugute. Dafür ein herzliches Dankschön! In dieser Rubrik informie- ren wir Sie regelmäßig über neue Leitlinien und Leitlinien-Updates.

\section{Neurosyphilis: Leitlinie komplett überarbeitet}

Die einstige Massenerkrankung Syphilis tritt in Deutschland wieder häufiger auf. Im Jahr 2018 erreichte die Zahl der Neuinfektionen mit 7332 Fällen einen neuen Höchstwert. Aus diesem Anlass hat die Deutsche Gesellschaft für Neurologie (DGN) die S1-Leitlinie "Neurosyphilis“ (AWMFRegisternummer 030/101) neu aufgelegt. Prof. Matthias Klein, München, und Prof. Jörg Weber, Klagenfurt, haben die Leitlinienarbeit koordiniert.

Die S1-Leitlinie, die bis Mai 2025 gültig ist, richtet sich an Neurologen, Psychiater, Internisten, Infektiologen und Ärzte, die Patienten mit HIV im stationären und ambulanten Bereich be- 


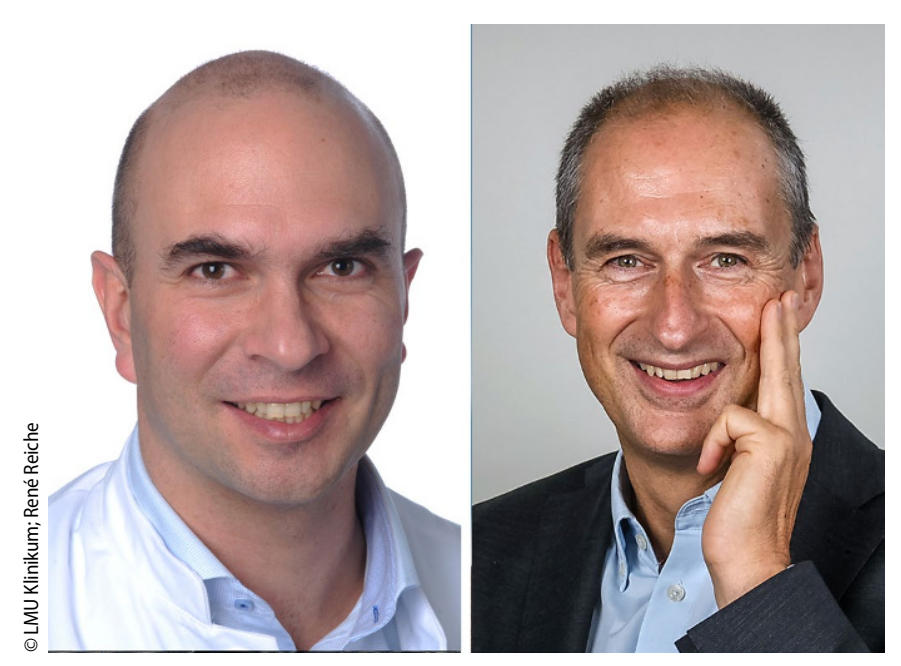

$\Delta$ V. I. n. r.: Prof. Matthias Klein, München, und Prof. Jörg Weber, Klagenfurt

handeln. Sie beschreibt klinische Verlaufsformen und diagnostische Kriterien und gibt Empfehlungen zu Behandlung und Verlaufskontrolle der verschiedenen Manifestationsformen der Neurosyphilis.

Neurologische Manifestationsformen der Syphilis können nach der Primärsyphilis in jedem Stadium auftreten. Klinische Hauptmanifestationsformen der „frühen Neurosyphilis“ (wenige Monate bis Jahre nach Infektion) sind eine Meningitis und etwas später eine meningovaskuläre Manifestation. Zudem kann es zu Granulomen, sogenannten syphilitischen Gummen, kommen. Die „späte Neurosyphilis“ manifestiert sich Jahre und Jahrzehnte nach der Infektion meist als paralytische oder als tabische Neurosyphilis.

\section{Penicillin G ist Therapie erster Wahl}

Der Verdacht auf Neurosyphilis ergibt sich entweder aus neurologischen oder psychiatrischen

\section{Neue EAN-Leitlinie zum Medikamentenübergebrauchs- kopfschmerz}

Die European Academy of Neurology (EAN) hat unter Federführung von DGN-Pressesprecher Professor Dr. Hans-Christoph Diener, Essen, eine Leitlinie zum Management des Medikamentenübergebrauchskopfschmerzes publiziert. Sie gibt Präventions- und Therapieempfehlungen, wie der Teufelskreis zwischen Kopfschmerzen und Einnahme von Schmerzmedikamenten und Migränemitteln vermieden bzw. durchbrochen werden kann.

Symptomen plus anamnestischen Angaben über eine frühere Geschlechtskrankheit und/oder eine positive Treponemenserologie. Personen mit HIV haben ein erhöhtes Risiko für eine Neurosyphilis. Diagnostisch kann man zwischen einer wahrscheinlichen und einer sicheren Neurosyphilis unterscheiden. Eine Herausforderung ist es, asymptomatische HIV-positive Patienten mit Neurosyphilis zu erkennen und frühzeitig eine Liquoruntersuchung zu initiieren; hier gibt die Leitlinie klare Empfehlungen.

Die Therapie der ersten Wahl besteht in der intravenösen Gabe von Penicillin G in hoher Dosis über 14 Tage. Alternativ kann auch mit $1 \times 2 \mathrm{~g} /$ Tag Ceftriaxon intravenös über 14 Tage behandelt werden. Eine klinische Besserung und ein Rückgang der Liquorparameter unter der antibiotischen Therapie zeigen das Ansprechen auf die Behandlung. Die Leitlinienautoren empfehlen Verlaufsuntersuchungen des Liquors drei bis sechs Monate nach Behandlung. ckeln, sind Patienten, die an einer weiteren Schmerzerkrankung leiden, wie z.B. chronischen Rückenschmerzen, oder Menschen mit schwerer Migräne; häufige Begleiterkrankungen des $\mathrm{MOH}$ sind Angsterkrankung und Depression.

Die neue Leitlinie der European Academy of Neurology gibt anhand von sieben sogenannten $\mathrm{PICO}$ questions $(\mathrm{P}=$ popular, $\mathrm{I}=$ intervention, $\mathrm{C}=$ control, $\mathrm{O}=$ outcome) Empfehlungen für das Management von Medikamentenübergebrauchskopfschmerz. Grundlegende und wichtigste Präventionsmaßnahmen sind nach Ansicht der Autoren Information und Patientenedukation. Sie können maßgeblich dazu beitragen, dem $\mathrm{MOH}$ bei Migränepatienten vorzubeugen. Die Leitlinie empfiehlt darüber hinaus, dass „MOH-Risikopatienten“ in regelmäßigen Abständen (alle drei bis sechs Monate) vom Allgemeinmediziner oder Neurologen gesehen werden sollen. Diese Empfehlung ist zwar, streng genommen, nicht evidenzbasiert, laut Professor Dr. Hans-Christoph Diener, DGNPressesprecher und Erstautor der Leitlinie, aber eine "Common Sense“-Empfehlung. „Wir wissen, dass Patienten seltener

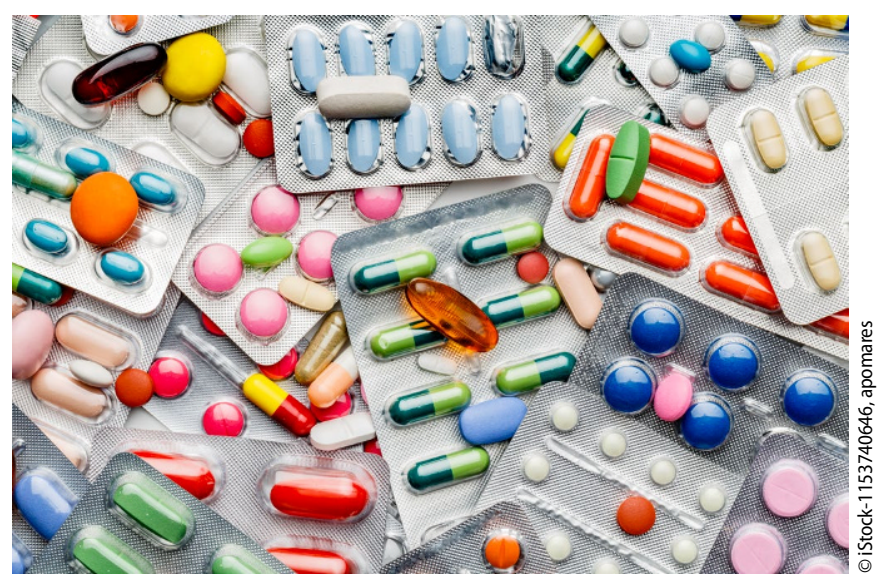


einen $\mathrm{MOH}$ entwickeln, die umfassend über den Zusammenhang von Schmerzmitteln und Schmerzmittelübergebrauchskopfschmerz informiert wurden, und es gibt Studien, die zeigen, dass ein Beratungsgespräch plus Print-Informationsmaterial um einiges effektiver ist als das Informationsmaterial allein. Es liegt auf der Hand, dass regelmäßige Gespräche die Sensibilität für die Thematik erhöhen und die Bereitschaft, trotz Schmerzen gelegentlich auf Medikamente zu verzichten oder die Dosis zu reduzieren, weiter stärken“, so der Experte.

Die intensive Beratung ist somit ein probates Mittel zur $\mathrm{MOH}$-Prävention, gelangt aber an Grenzen, wenn es um die $\mathrm{MOH}$ Behandlung geht: Die alleinige Beratung kann zwar bei Übergebrauch von Triptanen oder einfachen Analgetika zielführend sein, wenn keine größeren psychiatrischen Komorbiditäten vorliegen bei Übergebrauch von Opioiden, Barbituraten oder Tranquilizern rät die Leitlinie aber zur Überweisung an einen Kopfschmerzexperten oder in ein spezialisiertes Schmerzzentrum. Denn grundsätzlich muss immer ein Entzug oder zumindest eine sanfte Reduzierung der Übergebrauchsmedikamente erfolgen, um den $\mathrm{MOH}$ langfristig zu therapieren. Ein erfolgreiches Ausschleichen oder Absetzen der Schmerzmedikation gelingt fast nur in sehr enger Betreuung, die je nach Komplexität und Zustand des Patienten stationär, teilstationär oder ambulant erfolgen kann. Wichtig ist jedoch, dass diese Betreuung multidisziplinär erfolgt, neben Neurologen sollten auch Schmerzmediziner und Verhaltenspsychologen eingebunden sein.

Offen bleibt letztlich die Frage, zu welchem Zeitpunkt bei Patienten mit $\mathrm{MOH}$ und chronischer
Migräne eine gezielte Migränetherapie, z. B. durch Onabotulinumtoxin Typ A oder CGRPAntikörper, erfolgen sollte. „Im Prinzip ist es ratsam, die Patienten zunächst vom Schmerzmittelübergebrauch zu entwöhnen, bevor man diese spezifischen Migränemittel einsetzt, auch um beurteilen zu können, wie stark und häufig die Kopfschmerzen sind, wenn der $\mathrm{MOH}$ wegfällt. Auf der anderen Seite darf man nicht vergessen, dass gerade $\mathrm{Pa}$ tienten mit chronischer Migräne stark leidgeprüft sind und wir ihnen eine wirksame Medikation nicht über eine längere Zeit vorenthalten sollten. Die Entscheidung, wann die Migränetherapie initiiert wird, ist also immer nur individuell zu treffen“, so Diener.

„Die Bedeutung der vorliegenden Leitlinie liegt darin, dass sie auf das Problem des $\mathrm{MOH}$ aufmerksam macht und auch Ärztinnen und Ärzte für das Phänomen sensibilisiert. Bei einer Prävalenz von $70 \%$ bei Patienten mit chronischen Kopfschmerzen, das sind geschätzt über eine halbe Million Menschen, ist die $\mathrm{MOH}$ ein relevantes Gesundheitsproblem, das eine gesamtgesellschaftliche Aufmerksamkeit erfahren sollte. Die europäische Leitlinie unter der Federführung von Prof. Diener trägt dazu bei und wird hoffentlich vielen Betroffenen zur Schmerzfreiheit oder zumindest zu einer deutlichen Verbesserung der Kopfschmerzen verhelfen", kommentiert Professor Dr. Peter Berlit, Essen, Generalsekretär der DGN, abschließend.

\section{Literatur}

\section{HC Diener, F Antonaci, M Braschinsky et al (2020) Euro- unter: www.dgn.org/rubrik-dgn/ ausschreibungen-und-preise} pean Academy of Neurology guideline on the management of medication-overuse headache. European Journal of Neurology 2020. https://doi.org/10.1111/ene.14268 pisierungs-Plattform zur Untersuchung der ZNS/PNS-Beteiligung bei SARS-CoV-2.

Um eine mögliche Beteiligung des zentralen und peripheren Nervensystems bei COVID-19 systematisch zu untersuchen, wird derzeit ein deutschlandweites Register mit dezentralem, standardisiertem Sampling und Biobanking von humanen Gewebeproben aus definierten ZNS/PNS/ Muskel-Arealen bei COVID19-Obduktionen aufgebaut.

Es soll der wissenschaftlichen Gemeinschaft zur Verfügung gestellt und mit vorhandenen klinischen Patientenregistern vernetzt werden.
Standortübergreifende Phänoty-

\section{Ausschreibungen}

\section{ZNS/PNS-Beteiligung bei SARS-CoV-2}

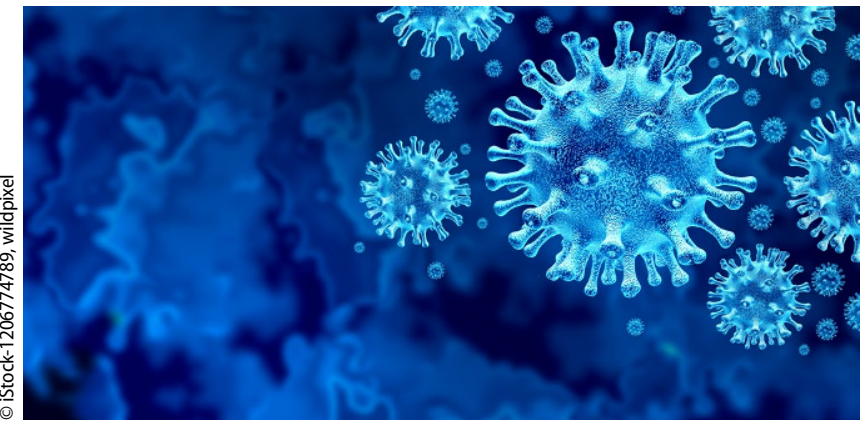

Das Projekt wurde von der Deutschen Gesellschaft für Neuropathologie und Neuroanatomie (DGNN) initiiert, die im Rahmen des Nationalen Forschungsnetzwerks COVID-19 der Universitätsmedizin eine standortübergreifende Phänotypisierungs-Plattform zur Untersuchung der ZNS/PNS-Beteiligung bei SARS-CoV-2 beantragt hat. Das erlaubt eine detaillierte morphologische, molekulare und klinische Charakterisierung von potenziellen ZNS/PNS-Infektionen bzw. Dysfunktionen durch SARS-CoV-2.

Das Projekt wird aktiv von der Deutschen Gesellschaft für Neurologie (DGN) unterstützt.
Aktuelle Ausschreibungen der DGN und anderer Organisationen sowie weitere Informationen finden Sie

\section{Nachwuchsförderung 2020 der Deutschen} Gesellschaft für Parkinson und Bewegungsstörungen e.V.

Die Deutsche Gesellschaft für Parkinson und Bewegungsstörungen e. V. verfolgt als wissenschaftliche Fachgesellschaft das
Ziel, die Diagnose, Prävention und Behandlung der ParkinsonKrankheit und anderer Bewegungsstörungen $\mathrm{zu}$ verbessern. Um die Forschung auf diesen Gebieten nachhaltig zu fördern und durch innovative Ideen $\mathrm{zu}$ beleben, schreibt die Gesellschaft zum fünften Mal eine Förderung von Forschungsprojekten speziell für Nachwuchswissenschaftlerinnen/-wissenschaftler aus. Im Rahmen dieser Ausschreibung werden zwei Forschungsprojekte gefördert. Es werden zweimal je 50.000 Euro zur Verfügung ge- 
stellt, die Wissenschaftlerinnen/ Wissenschaftler bis zum 40 . Lebensjahr beantragen können, die im Bereich der ParkinsonKrankheit und anderer Bewegungsstörungen zu klinischen und grundlagenorientierten Themen forschen. Bewerbungen können ab sofort bis einschließlich 15. September 2020 bei der Deutschen Gesellschaft für Parkinson und Bewegungsstörungen eingereicht werden. Bitte richten Sie Ihre Bewerbung ausschließlich in digitaler Form an folgende Adresse: nachwuchsförderung@ parkinson-gesellschaft.de.

Weitere Informationen finden Sie unter https://www.parkinsongesellschaft.de/

\section{Wissenschaftspreis 2020 der Deutschen Gesell- schaft für Parkinson und Bewegungsstörungen e.V.}

Die Deutsche Gesellschaft für Parkinson und Bewegungsstörungen e.V. schreibt zum sechsten Mal den Wissenschaftspreis „Neurodegenerative Erkrankungen“ aus. Der Preis prämiert vier hervorragende abgeschlossene wissenschaftliche Forschungsarbeiten zur Ätiologie, Pathogenese, Diagnostik, Therapie oder Versorgung neurodegenerativer Erkrankungen. Die vier Preise sind jeweils mit 2500 Euro dotiert. Wissenschaftlerinnen/ Wissenschaftler bis zum 40. Lebensjahr und Mitglieder der Arbeitsgruppen der DPG werden besonders zur Bewerbung aufgefordert. Wir bitten die Bewerberinnen/Berwerber, ihre Bewerbungsunterlagen bis einschließlich 15. September 2020 als PDF-Datei mit dem Betreff „Wissenschaftspreis" an folgende E-Mail-Adresse zu übersenden: wissenschaftspreis@parkinsongesellschaft.de.

Weitere Informationen finden Sie unter https://www.parkinsongesellschaft.de/

\section{Tuberöse Sklerose- Forschungspreis 2021}

Die Deutsche Tuberöse Sklerose Stiftung zeichnet Wissenschaftlerinnen und Wissenschaftler für innovative Leistungen auf dem Gebiet der Erforschung des Tuberöse Sklerose Complex (TSC) aus. Sie möchte hierdurch die weitere Erforschung des Krankheitsbildes in den Fokus der Öffentlichkeit rücken. Der Preis ist mit 10.000 Euro dotiert und steht dem/der Preisträger/in im Rahmen der Forschungsarbeiten zur freien Verfügung. Der Tuberöse Sklerose-Forschungspreis kann auch geteilt und an zwei Personen vergeben werden. $\mathrm{Da}$ bei können sowohl Projekte der Grundlagenforschung als auch solche mit direktem klinischem Bezug eingereicht werden. Bewerbungen sind in deutscher Sprache bis zum 15. September 2020 elektronisch (info@tsstiftung.org) an den Vorstand der Stiftung zu richten. https://www. ts-stiftung.org/

\section{Zi-Wissenschaftspreis „Regionalisierte Versorgungsforschung" 2020}

Das Zentralinstitut für die kassenärztliche Versorgung in Deutschland (Zi) schreibt 2020 erneut einen Wissenschaftspreis zur Förderung der Erforschung regionaler Unterschiede in der Gesundheitsversorgung aus. Mit dem Wissenschaftspreis werden überdurchschnittlich gute $\mathrm{Ar}$ beiten auf dem Gebiet der Versorgungsforschung ausgezeichnet, die sich der Untersuchung regionaler Unterschiede in der Gesundheitsversorgung widmen. Der Preis ist mit 7500 Euro dotiert und kann geteilt werden, sofern dies vom Entscheidungsgremium beschlossen wird Einsendeschluss für die Einreichung von Publikationen ist der 28. September 2020. Die Einsendung kann auf dem Postweg, per E-Mail oder persönlich erfolgen. Weitere Informationen finden Sie unter https://www.zi.de/

\section{Stipendium vom Verein Myelitis e.V.}

Der Verein Myelitis e.V. schreibt ein Stipendium für eine junge Neurologin oder einen jungen Neurologen aus, die/der sich insbesondere für die Transverse Myelitis und ähnliche seltene neuroimmunologische Erkrankungen interessiert. Das dreimonatige Stipendium (01.07.-30.09.2021) dient der Weiterbildung und findet im TM-Center des Johns Hopkins Hospital in Baltimore/ Maryland unter der Leitung von Professor Dr. Carlos Pardo-Villamizar statt. Die Stipendiatin/ der Stipendiat wird zum „Research Fellow" ernannt, der sowohl in der Forschung als auch in der klinischen Beobachtung tätig ist. Das Stipendium umfasst in den besagten drei Monaten Zahlungen für den Lebensunterhalt von ca. 3600 Euro/ Monat, Zahlungen für eine Unterkunft von ca. 1600 Euro/Monat und eine Auslandskrankenversicherung. Die Auswahl der Stipendiatin/des Stipendiaten soll bis spätestens 30. September 2020 abgeschlossen sein. Bewerbungen (Motivationsschreiben und Lebenslauf) und Rückfragen sind mit entsprechendem Vorlauf zu richten an: Pilar Schnadenberger (Telefon: 0157-365 77225; E-Mail: pilar@ myelitis.de). Weitere Informationen finden Sie unter http:// www.myelitis.de

\section{DGNI-Nachwuchsförde- rungspreis 2021}

Die Deutsche Gesellschaft für NeuroIntensiv- und Notfallmedizin schreibt in diesem Jahr wieder einen Preis zur Projektförderung von NachwuchswissenschaftlerInnen aus. Der Preis wird für innovative Forschungsprojekte in der NeuroIntensivmedizin verliehen, die sich mit den Zielen der Satzung gemäß $\$ 2$ decken. Das Preisgeld kann durch das Preiskuratorium aufgeteilt werden. Die Bewilligung der Förderung wird während der
Eröffnungsveranstaltung auf der ANIM vom 21.01.-23.01.2021 in Berlin in Form einer Urkunde verliehen und ist mit einer Geldprämie von bis zu 20.000 Euro verbunden. Die Preisträgerin/ der Preisträger ist Mitglied der DGNI und mit der Annahme des Preises zur schriftlichen und persönlichen Vorstellung der Forschungsergebnisse auf der ANIM verpflichtet. Bewerbungsunterlagen sind als PDF per E-Mail bis zum 30. September 2020 an die Schriftführerin der DGNI Dr. Katja Wartenberg zu richten: katja.wartenberg@medizin. unileipzig.de Weitere Informationen finden Sie unter https:// www.dgni.de/

\section{Wissenschaftspreis der Deutschen Gesellschaft für Liquordiagnostik und Klinische Neurochemie e.V. (DGLN)}

Der Preis wird von der Deutschen Gesellschaft für Liquordiagnostik und Klinische Neurochemie e. V. ausgeschrieben. Prämiert werden deutschsprachige klinisch-neurologisch und klinisch-chemisch tätige Wissenschaftlerinnen/Wissenschaftler für ein abgeschlossenes wissenschaftliches Projekt zum Thema Liquordiagnostik, das in den Jahren 2019 und 2020 zur $\mathrm{Pu}$ blikation eingereicht oder angenommen bzw. in den letzten 24 Monaten vor Ablauf der Bewerbungsfrist publiziert wurde.

Der Preis wird anlässlich der alle zwei Jahre stattfindenden Jahrestagung der DGLN in Form einer Urkunde überreicht und ist mit einer Geldprämie von 2000 Euro verbunden. Die Preisverleihung zur aktuellen Ausschreibung findet bei der Jahrestagung der DGLN in Erlangen (07.05.-08.05.2021) statt.

Den Unterlagen sind das Manuskript bzw. die Publikation, das Schriftenverzeichnis und der Lebenslauf beizulegen. Sie sind als PDF per E-Mail bis zum 31. Dezember 2020 zu richten an die Geschäftsstelle der DGLN e.V.: info@dgln.de 


\section{Umfrage der Heinrich-Heine-Universität Düssel- dorf zum Thema „Preise und Auszeichnungen in der Wissenschaft"}

Im Rahmen eines Forschungsprojekts über Forschungspreise in der Medizin hat die Uni Düsseldorf einen Fragebogen entwickelt. Das Ziel ist, mehr über die Funktionen von Forschungspreisen zu erfahren; die Neurologie bietet uns dafür ein sehr gutes Sample an. Der Deutschen Gesellschaft für Neurologie bringt der Fragebogen Erkenntnisse über die Verankerung ihrer traditionsreichen und neuen Auszeichnungen in der Fachgesellschaft und die damit zusammenhängende Forschungsförderung. Wissenschaftshistorisch sollen aus einem geplanten Vergleich mit weiteren Fachgesellschaften wie Chirurgen und Pharmakologen Gemeinsamkeiten, aber auch Unterschiede national und international herausgearbeitet werden.

Das Ausfüllen des Online-Fragebogens (https://ww3.unipark.de/uc/ Forschungspreis) dauert nur ca. 2-4 Minuten. Der Link ist bis zum 2. Oktober 2020 aktiv.

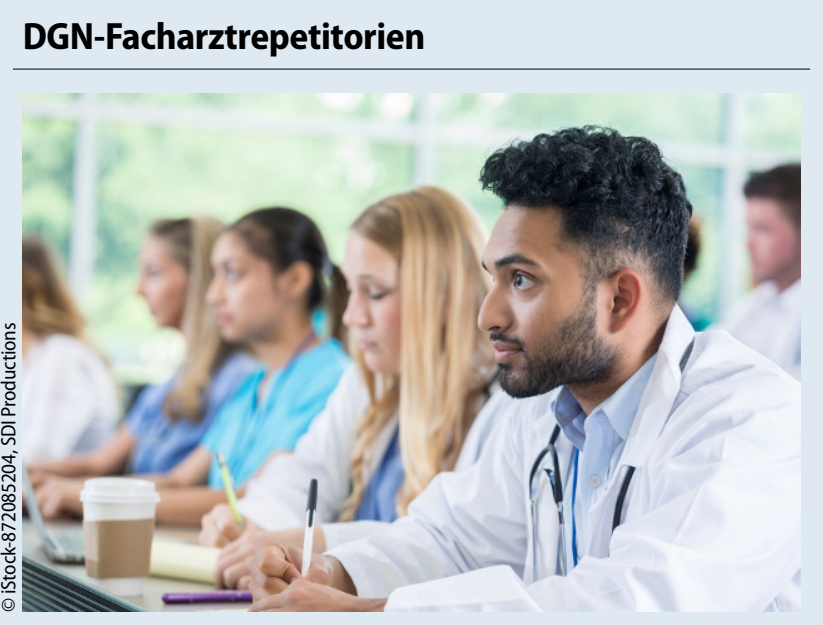

Das Facharztrepetitorium in Juist ist ausgebucht. Das nächste Facharztrepetitorium der DGN findet vom 04.-06. März 2021 in Essen statt.

\section{Fortbildung der Deutschen Gesellschaft für Neurowissenschaftliche Begutachtung e.V.}

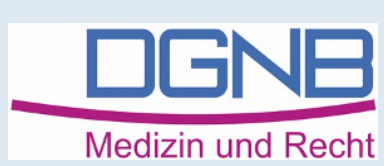

DGNB-Intensivseminar Medizinische Begutachtung/Seminarblock 1 Das Intensivseminar zur strukturierten curricularen Fortbildung Medizinische Begutachtung nach dem Curriculum der Bundesärztekammer beinhaltet 64 Unterrichtseinheiten. Diese werden in zwei Seminarblöcken zu je drei Tagen angeboten. Block 1 in Günzburg vom 02.-04.07.2020 und Block 2 in Kassel vom 15.-17.10.2020. Das Seminar wird ggf. als Webinar stattfinden.

Informationen dazu und zu allen anderen Veranstaltungen finden Sie unter www.dgnb-ev.de/veranstaltungen

\section{Bitte vormerken!}

Der 1. Supervisionskurs für Gutachten wurde auf den 6. Mai 2021 verschoben.

\section{Online-Fortbildung}

ERN-RND-Webinare zu seltenen Bewegungsstörungen und neuromuskulären Erkrankungen

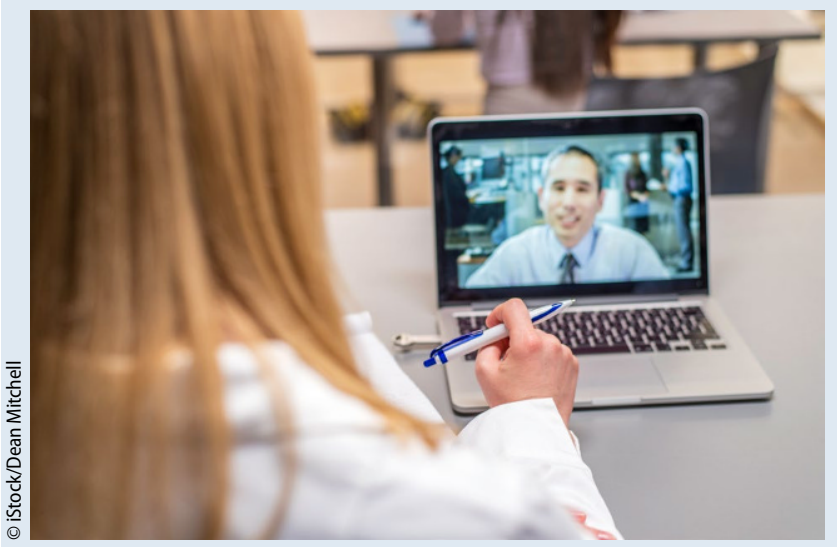

Um Neurologen europaweit im Bereich seltenere neurologische Bewegungsstörungen und neuromuskuläre Erkrankungen zu schulen, bietet die ERN-RND Webinare unter der Leitung europäischer Experten an.

Die Webinare wurden in Zusammenarbeit mit dem European Reference Network for Rare Neuromuscular Diseases (EURO-NMD) und der European Academy of Neurology (EAN) aufgelegt und werden von verschiedenen europäischen Experten durchgeführt. Es werden Erkrankungen sowohl des Kindes- und Jugendalters als auch des Erwachsenenalters thematisiert.

Eine Übersicht aller Termine finden Sie hier: http://www.ern-rnd.eu/ education-training/webinars 


\section{Save the Date}

Bitte informieren Sie sich, ob die aufgeführten Veranstaltungen wegen der aktuellen Einschränkungen durch die SARS-CoV-2-Pandemie wie geplant stattfinden können!

\section{Fortbildungen}

Intensivkurs Neurologie, 09.12. September 2020, Bad Segeberg

Refresherkurs - Spezielle Neurologische Ultraschalldiagnostik im September 2020, 28.-29. September 2020, Essen

Facharztrepetitorium 2020 in Dresden, 30. Sep.-02. Okt. 2020, Dresden

16. Stralsunder Neurophysiologie-Wochenende mit Technikseminar, 02.-04. Oktober 2020, Stralsund

14. DGNB-Refresherseminar Neurowissenschaftliche Begutachtung, 09.-10. Oktober 2020, Frankfurt am Main

23. Refresherseminar Farbduplexsonografie der hirnversorgenden Gefäße - spezielle neurologische Ultraschalldiagnostik, 10. Oktober 2020, München

DGNB-Intensivseminar zur strukturierten curricularen Fortbildung „Medizinische Begutachtung" nach dem Curriculum der Bundesärztekammer/Seminarblock 2, 15.-17. Oktober 2020, Kassel (siehe Infokasten)

\section{FEES-Basisseminare - Flexible endoskopische Evaluation des Schluckens}

14.-16. August 2020, Berlin

04.-06. September 2020, Berlin

17.-19. September 2020, Bochum

01.-03. Oktober 2020, Aachen

16.-18. Oktober 2020, Berlin

\section{FEES-Expertenworkshops}

26.-27. Oktober 2020, München

Weitere Informationen online: www.dgn.org/rubrik-themen/fees

\section{Kongresse und Symposien}

Jahrestagung der Sektion Wirbelsäule der Deutschen Gesellschaft für Neurochirurgie (DGNC) e. V., 04.-05. September 2020, Mainz, https://www. sektionstagung-ws.de/

55. Jahrestagung der Deutschen Gesellschaft für Epileptologie (DGfE), neuer Termin: 06.09. September 2020, Freiburg, https://www.epilepsie-tagung.de

35. Jahrestagung der Gesellschaft für Neuropsychologie, 25-26. September 2020: Digitaler Kongress, weitere Informationen unter https://www.gnp.de

Sektionstagung Periphere Nerven der Deutschen Gesellschaft für Neurochirurgie (DGNC) e. V., 25.-26. September 2020, Freiburg, https://www. sektionstagung-pn.de/

11th World Congress for Neurorehabilitation, 07.-10. Oktober 2020 , Lyon

Eurospine 2020, 07.-09. Oktober 2020, Digitaler Kongress, https:// www.eurospinemeeting.org/vienna2020

Jahrestagung Sektion Neuroonkologie der Deutschen Gesellschaft für Neurochirurgie (DGNC) e. V., 16.-17. Oktober 2020, Berlin, https://www. sektionstagung-no.de

Süddeutscher Neurochirurgentag 2020, 17. Oktober 2020, Ravensburg

Weitere Informationen online: www.dgn.org/fortbildungskalender

\section{Personalia}

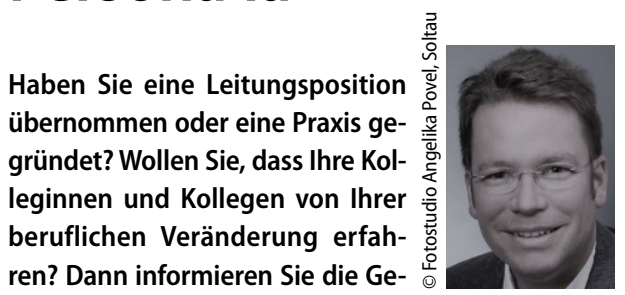
schäftsstelle der DGN unter der EMail-Adresse: info@dgn.org.

\section{In den Ruhestand verab- schiedet}

Dr. Kirsten Jacobs-Greve, vorher im MVZ Neurologie Helios Klinik Schleswig tätig, ist seit dem 01.07.2020 berentet. Sie ist DGNMitglied seit 01.01.2000.

\section{Berufliche Veränderungen}

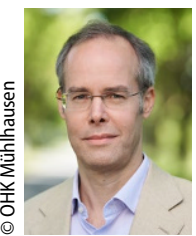

Dr. Oliver Freitag, vorher Oberarzt der Psychiatrischen Klinik der Kliniken Essen Mitte, ist seit dem 01.06.2020 Chefarzt der Abteilung für Gerontopsychiatrie des Ökumenischen Hainich Klinikums Mühlhausen. Er ist DGN-Mitglied seit 01.01.2006.

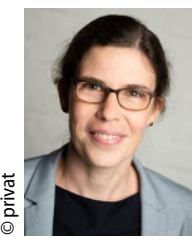

PD Dr. Annette Spreer, vorher leitende Oberärztin an der Universitätsmedizin Mainz, ist seit dem 01.05.2020 Chefärztin der neurologischen Klinik des Städtischen Klinikums Braunschweig. Sie ist DGN-Mitglied seit 01.02.2002.
Dr. Karsten Meyer-Wiethe, MHBA, vorher Oberarzt im MediClin Klinikum Soltau, ist seit dem 01.07.2020 niedergelassen in Geesthacht. Er ist DGN-Mitglied seit 01.01.2001.

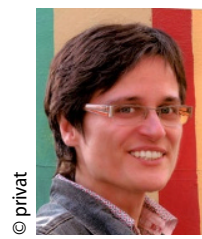

Dr. Elisabeth Keller, vorher Oberärztin im Hegau-Jugendwerk Gailingen, ist seit dem 01.07.2020 Oberärztin im Zentrum für Psychosomatik Zürich des Sanatoriums Kilchberg in der Schweiz. Sie ist DGN-Mitglied seit 01.01.1998.

Dr. Vera Schrader, bisher niedergelassene Ärztin in Porta Westfalica, hat ihre Praxis an Frau Dr. Astrid Beyerle-Inderfurth am 01.07.2020 übergeben und ist nun im Angestelltenverhältnis dort tätig. Sie ist DGN-Mitglied seit 01.01.1996. 


\section{DGN' Neurojobs}

\section{Aktuelle Jobangebote}

\section{Chefarztpositionen}

21318 Klinikum Lüneburg, Chefarzt für Neurologie $(\mathrm{m} / \mathrm{w} / \mathrm{d})$ 28325 Klinikum Bremen Ost, Chefarzt für Neurologie $(\mathrm{m} / \mathrm{w} / \mathrm{d})$ 29525 Helios Klinikum Uelzen, Chefarzt für Neurologie $(\mathrm{m} / \mathrm{w} / \mathrm{d})$ 46242 Knappschaftskrankenhaus Bottrop, Chefarzt für Neurologie $(\mathrm{m} / \mathrm{w} / \mathrm{d})$

78262 Hegau Jugendwerk Gailingen, Chefarzt für Neurologie $(\mathrm{m} / \mathrm{w} / \mathrm{d})$

\section{Schweiz:}

8008 Klinik Lengg, Zürich (CH), Medizinischer Direktor Epileptologie $(\mathrm{m} / \mathrm{w} / \mathrm{d})$

\section{Oberarztpositionen}

19395 Mediclin Krankenhaus Plau am See, Oberarzt (m/w/d) 27574 Klinikum BremerhavenReinkenheide, Ltd. Oberarzt (m/w/d)

29525 Helios Klinikum Uelzen, Oberarzt Neurologie $(\mathrm{m} / \mathrm{w} / \mathrm{d})$

38118 Klinikum Braunschweig, Oberarzt (m/w/d)

38723 Asklepios Kliniken Schildautal Seesen, Oberarzt $(\mathrm{m} / \mathrm{w} / \mathrm{d})$

44309 Knappschaftskrankenhaus, Dortmund, Oberarzt $(\mathrm{m} / \mathrm{w} / \mathrm{d})$

49152 Dr. Becker Neurozentrum Niedersachsen, Bad Essen, Ober$\operatorname{arzt}(\mathrm{m} / \mathrm{w} / \mathrm{d})$

49401 Krankenhaus St. Elisabeth Damme, Oberarzt (m/w/d)

57627 DRK Kamillus Klinik Asbach, Oberarzt (m/w/d)

59755 Klinikum Hochsauerland, St. Johannes-Hospital, ArnsbergNeheim, Oberarzt (m/w/d) 72488 SRH Krankenhaus Sigmaringen, Oberarzt $(\mathrm{m} / \mathrm{w} / \mathrm{d})$ 91056 Bezirkskliniken Mittelfranken - Erlangen, Oberarzt (m/w/d)

91522 Bezirksklinikum Ansbach, Oberarzt (m/w/d) 99437 Zentralklinik Bad Berka, Oberarzt $(\mathrm{m} / \mathrm{w} / \mathrm{d})$

\section{Facharztpositionen}

12683 Unfallkrankenhaus Berlin, Facharzt Neurologie (m/w/d) 22087 Marienkrankenhaus Hamburg, Facharzt Neurologie $(\mathrm{m} / \mathrm{w} / \mathrm{d})$

38118 Klinikum Braunschweig, Facharzt Neurologie (m/w/d) 40670 St. Mauritius Therapieklinik Meerbusch Düsseldorf, Facharzt Neurologie (m/w/d) 44309 Knappschaftskrankenhaus Dortmund, Facharzt Neurologie $(\mathrm{m} / \mathrm{w} / \mathrm{d})$

48149 Universitätsklinikum Münster, Facharzt Neurologie (m/w/d)

49152 Dr. Becker Neurozentrum Niedersachsen, Bad Essen, Facharzt Neurologie (m/w/d) 56068 Therapiezentrum Koblenz, Facharzt Neurologie $(\mathrm{m} / \mathrm{w} / \mathrm{d})$ 67655 Westpfalz-Klinikum Kaiserslautern, Facharzt Neurologie $(\mathrm{m} / \mathrm{w} / \mathrm{d})$

70199 Marienhospital Stuttgart, Facharzt Neurologie $(\mathrm{m} / \mathrm{w} / \mathrm{d})$

71364 Rems-Murr-Kliniken Winnenden, Facharzt Neurologie $(\mathrm{m} / \mathrm{w} / \mathrm{d})$

88048 Medizin Campus Bodensee Friedrichshafen, Facharzt Neurologie $(\mathrm{m} / \mathrm{w} / \mathrm{d})$

Diakonische Einrichtung in NRW, Facharzt Neurologie $(\mathrm{m} / \mathrm{w} / \mathrm{d})$

Frankreich:

75013 Brain and Spine Institute Paris/FR, Facharzt Neurologie $(\mathrm{m} / \mathrm{w} / \mathrm{d})$

\section{Ärzte in Weiterbildung -} Positionen für Assistenzärzte $(\mathrm{m} / \mathrm{w} / \mathrm{d})$ in der Neurologie 01307 Universitätsklinikum Carl Gustav Carus Dresden

04552 Sana Klinikum Leipziger Land, Borna

15236 Klinikum Frankfurt (Oder)

18435 Helios Hanseklinikum

Stralsund

22087 Marienkrankenhaus Hamburg

22417 Asklepios Klinik Nord Hamburg
23562 UKSH Campus Lübeck 14768 imland Klinik Rendsburg 25524 Klinikum Itzehoe 27574 Klinikum BremerhavenReinkenheide 35392 Universitätsklinikum Gie-

ßen und Marburg 38118 Klinikum Braunschweig 39130 Klinikum Magdeburg 40670 St. Mauritius Therapieklinik Meerbusch Düsseldorf 42697 St. Lukas Klinik Solingen 44309 Knappschaftskrankenhaus Dortmund 45131 Alfried Krupp Krankenhaus Essen-Rüttenscheid 45355 Katholisches Klinikum Essen

45964 St. Barbara Hospital in Gladbeck

47906 Hospital zum Heiligen Geist Kempen

49152 Dr. Becker Neurozentrum

Niedersachsen, Bad Essen

49401 Krankenhaus St. Elisabeth

Damme

53567 DRK Kamillus Klinik Asbach

53879 Marien-Hospital Euskirchen

63069 Sana Klinikum Offenbach 64283 Klinikum Darmstadt
66740 DRK-Krankenhaus Saarlouis

67655 Westpfalz Klinikum Kaiserslautern

70199 Marienhospital Stuttgart

71364 Rems-Murr-Kliniken

Winnenden

77933 Ortenau Klinikum LahrEttenheim

78476 Kliniken Schmieder 79539 Kliniken des Landkreises

Lörrach

80804 Schön Klinik München Schwabing

83670 m\&i Fachklinik Bad Heilbrunn

87600 Bezirkskliniken Schwaben

- Kaufbeuren

88048 Campus Bodensee Friedrichshafen

88212 Oberschwabenklinik St. Elisabethen-Klinikum Ravensburg

96450 REGIOMED Klinikum

Coburg

97422 Leopoldina Krankenhaus Schweinfurt

(Stand 02.07.2020)

Weitere Jobangebote finden Sie tagesaktuell online auf www.dgn-neurojobs.org 